\title{
Functionalization of polymerized 3D microstructures for biological applications
}

\author{
Ph.D. Thesis
}

Aekbote L Badri Prashad

Supervisor: Dr. Lóránd Kelemen

Institute of Biophysics, Biological Research Centre Hungarian Academy of Sciences

Szeged

2015 


\section{Table of Contents}

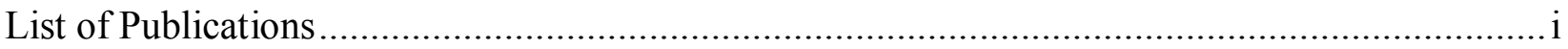

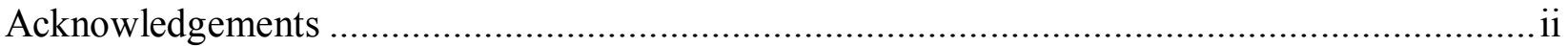

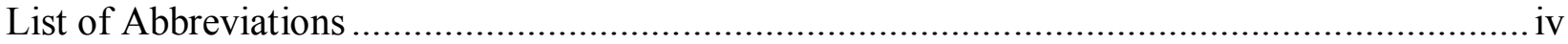

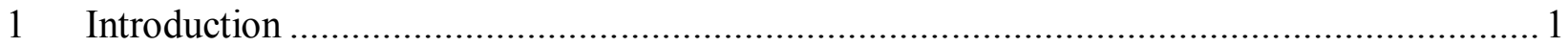

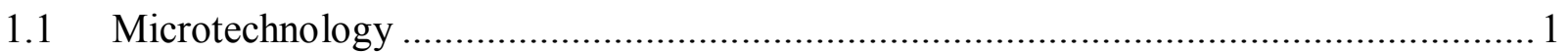

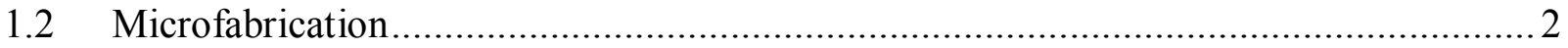

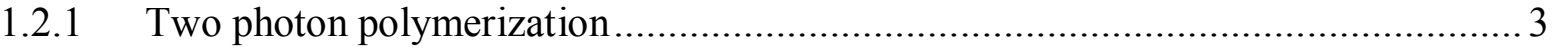

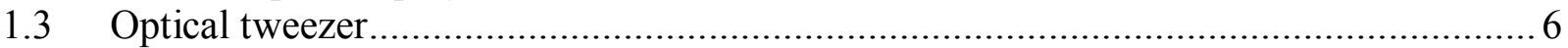

1.3.1 Measuring forces with optical tweezers …….................................................. 7

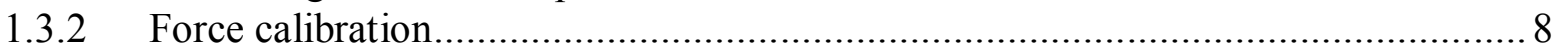

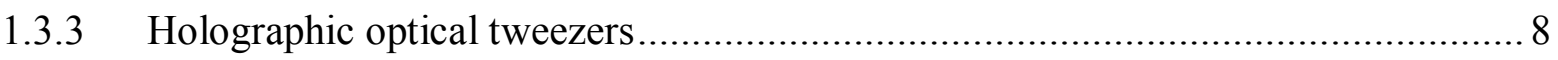

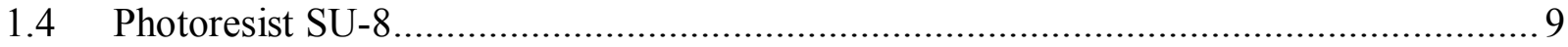

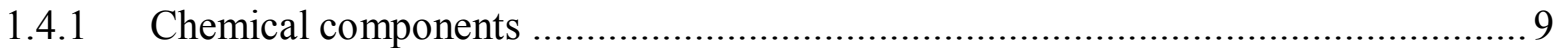

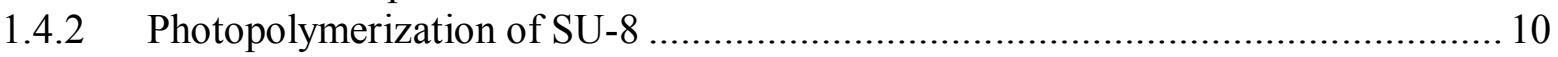

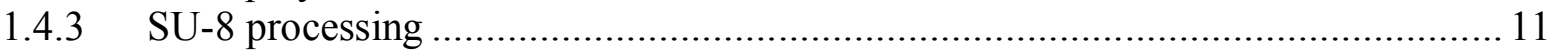

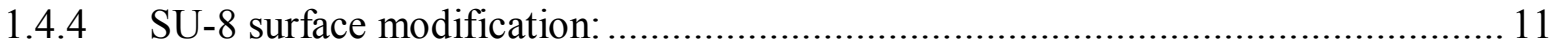

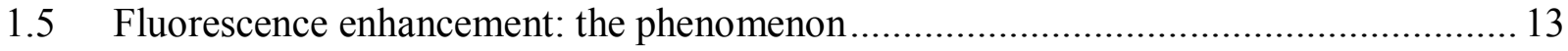

1.5.1 Principle of metal fluorescence enhancement..................................................... 14

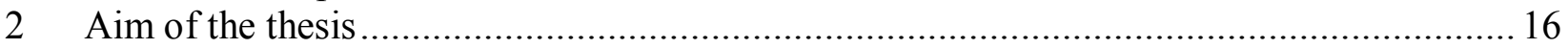

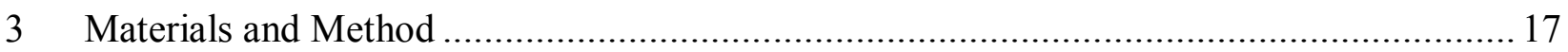

3.1 Fabrication of SU-8 layer by UV lithography .......................................................... 17

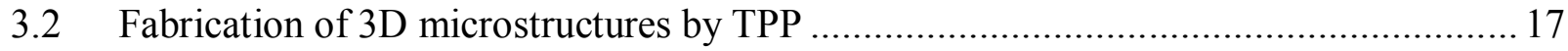

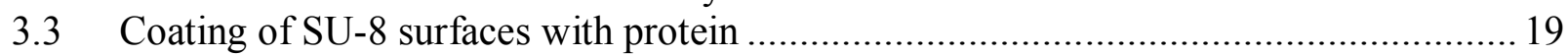

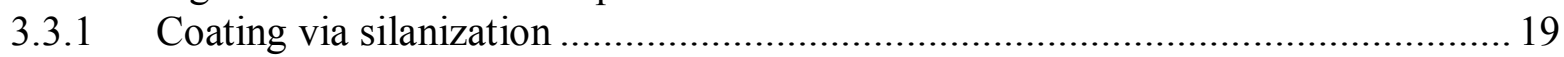

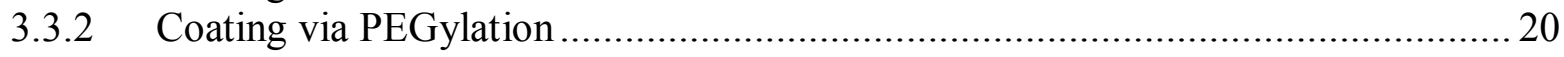

3.4 Preparation of a fluorescent protein layer on glass coverslip ..................................... 21

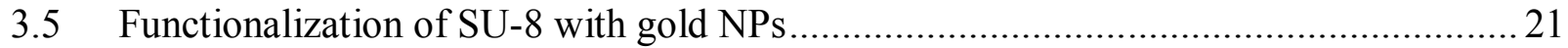

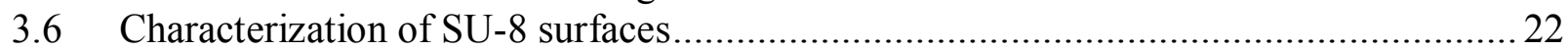

3.7 Fluorescence enhancement and reflectivity measurements .....................................2. 24

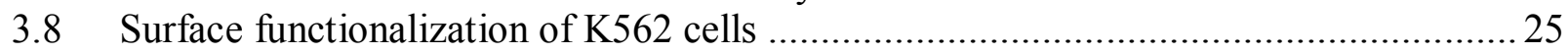

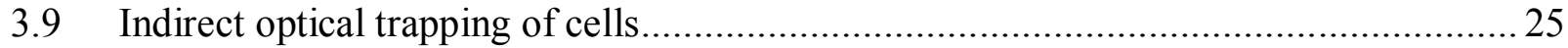

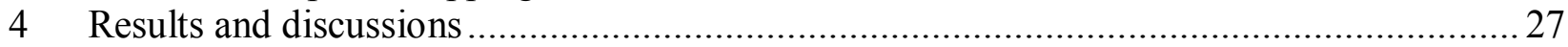

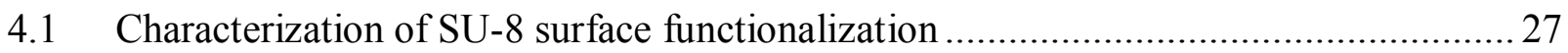

4.1.1 Evaluation of the residual epoxy groups in SU-8 layers ....................................2 27

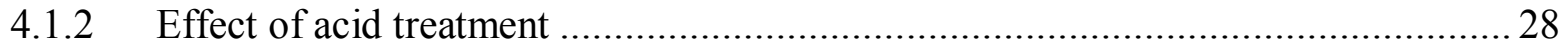

4.1.3 Quantification of protein density on SU-8 surface ..............................................2 29

4.1.4 Characterization of gold NPs on SU-8 layers and microstructures.......................... 32

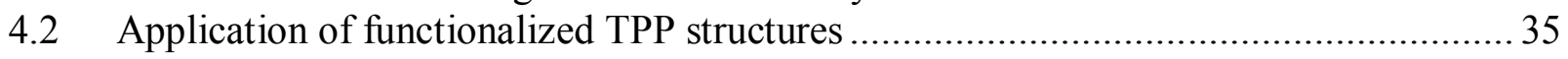

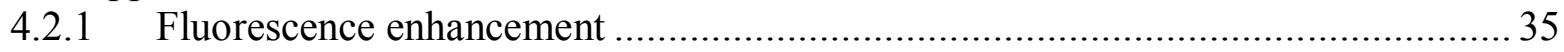

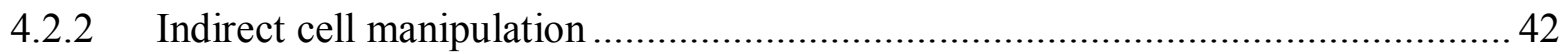

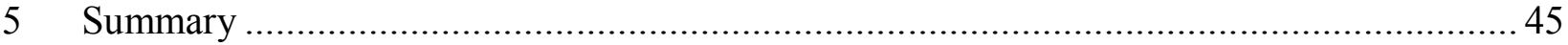

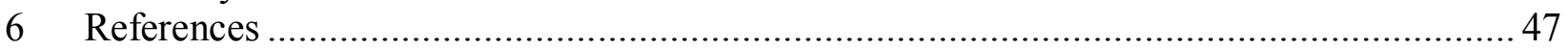




\section{List of Publications}

\section{Articles related to the subject of the thesis}

I. Aekbote B L, Jacak J, Schütz G J, Csányi E, Szegletes Z, Ormos P, Kelemen L. Aminosilane-based functionalization of two-photon polymerized 3D SU-8 microstructures. European Polymer J 48 (2012) 1745-1754. IF:2.562

II. Aekbote B L, Ormos P, Kelemen L. Gold nanoparticle-mediated fluorescence enhancement by two-photon polymerized 3D microstructures. Optical Materials 38 (2014) 301-309. IF:2.075

\section{Conference proceeding related to the thesis}

I. Aekbote B L, Kelemen L, Buzas A, Ormos P. Optical Tools For Localized Fluorescence Enhancement and Single Cell Studies. European Biophysics Journal with Biophysics Letters 42, (2013) S139-S139. IF: 2.47

\section{Conference proceedings and manuscript not related to the thesis}

I. Vizsnyiczai G, Kelemen L, Aekbote B L, Buzas A, Ormos P. Indirect optical manipulation of live cells with functionalized polymer microtools. European Biophysics Journal with Biophysics Letters 42, (2013), S114-S114. IF: 2.47

II. Kelemen L, Aekbote B L, Buzas A, Ormos P .Microtools made by two-photon polymerization for optical tweezers systems used in biological manipulation experiments. European Biophysics Journal with Biophysics Letters 40, (2011), 228. IF: 2.1

III. Vizsnyiczai G, Lestyán T, Joniova J, Aekbote B L, Ormos P, Miskovsky P, Kelemen L, Bánó G. Optically trapped surface-enhanced Raman probes prepared by silver photoreduction to 3D microstructures (under Review). 


\section{Acknowledgements}

This thesis would not have been possible without the help of many in so many ways, mainly in the Institute of Biophysics, Biological Research Centre of the Hungarian Academy of Sciences.

First and foremost I thank God for being my strength and guide throughout these years. Without Him, I would not have had the wisdom or the physical ability to come thus far.

I am forever grateful to Dr. Lóránd Kelemen, my supervisor who introduced me to the subject of micro and nanotechnology. His constant patience, guidance and effort were essential throughout my $\mathrm{PhD}$ work. He has gone beyond the call of a thesis advisor to assume the role of a academic colleague and friend, making an effort to not only explain the concepts of various aspects of this thesis but to also creating a stimulating atmosphere and giving interesting feedbacks and valuable discussions. Additionally his guidance developed my experimental skills and our everyday debates about the projects broadened my knowledge and made my work and life easier.

I would like to convey my heartfelt gratitude and sincere appreciation to Prof. Pál Ormos, the director of the Institute of Biophysics for giving me the opportunity to do my $\mathrm{PhD}$ in his group, I am truly grateful for his timely support and guidance. Without his help and orientation this thesis would not have been possible.

I also want to convey my deepest gratitude to my colleagues, Gaszton Vizsnyiczai, Dr. Sándor Valkai, András Buzás and Dr. Zsolt Szegletes for their invaluable discussions help and support.

I am also deeply thankful to the technical staff Kissné Domonkos Mariá, Izabella Egri and the administrative staff Anikó Hrk, Beáta Verebes and Zsófia Melczer.

I wish to acknowledge our collaborator Prof. Schütz G J and Dr. Jacak J, from the Institute of Applied Physics, Vienna University of Technology,Vienna, and Biophysics Institute, Johannes Kepler University Linz, Austria respectively for their help in quantification of protein density . I am also thankful to Dr. Félix Schubert, University of Szeged, Department of Mineralogy, Geochemistry and Petrology, Szeged for his help in microspectroscopy. 
I also thank the support in different way of my extended Church family friends, my cousin brother and sisters, my ITC friends, my friends Dr. Sajith O T, Dr. Hima Bindu Gali, Dr. Yathish A J, the small Indian community and many more here in Szeged.

The completion of this thesis could not have been accomplished without my Parents constant support and motivation throughout my life and during my $\mathrm{PhD}$ work here. This thesis is heartily dedicated to them and to my caring, loving, and supportive wife Dr. Joseph Mary Prathiba for her daily scientific encouragement and prayers, without her I could not have finished this thesis, and finally to my loving daughter Nivedya Badri Aekbote who brought new hope and joy to complete this thesis. 


\section{List of Abbreviations}

\begin{tabular}{|c|c|}
\hline AFM & Atomic Force Microscopy \\
\hline APTES & 3-AminoPropyl TriEthoxySilane \\
\hline $\mathrm{BaF}_{2}$ & Barium Fluoride \\
\hline Bio-MEMS & Biomedical- MicroElectroMechanical Systems \\
\hline BSA & Bovine Serum Albumin \\
\hline bBSA & Biotinylated Bovine Serum Albumin \\
\hline CAN & Ceric-Ammonium Nitrate \\
\hline CCD & Charge Coupled Device \\
\hline DOE & Diffractive Optical Element \\
\hline FLIC & Fluorescence Interference Contrast Microscopy \\
\hline FTIR & Fourier Transform Infrared Spectroscopy \\
\hline GBL & Gamma-ButyroLactone \\
\hline HEPES & 4-(2-HydroxyEthyl)-1-PiperazineEthaneSulfonic acid \\
\hline HOT & Holographic Optical Tweezer \\
\hline IgG & Immunoglobulin $\mathrm{G}$ \\
\hline IR & Infra-Red \\
\hline LED & Light-Emitting Diode \\
\hline LSCM & Laser Scanning Confocal Microscope \\
\hline LSPR & Localized Surface Plasmon Resonance \\
\hline MEF & Metal Enhance Fluorescence \\
\hline MEMS & MicroElectroMechanical Systems \\
\hline Mthd & Method \\
\hline NA & Numerical Aperture \\
\hline NIR & Near Infra-Red \\
\hline NPs & NanoParticles \\
\hline ORMOCER & ORganic MOdified CERamics \\
\hline OT & Optical Tweezer \\
\hline PBS & Phosphate-Buffered Saline \\
\hline PEB & Post Exposure Bake \\
\hline PEG & Poly(Ethylene Glycol) \\
\hline PEGDA & Poly(Ethylene Glycol) DiAcrylate \\
\hline PDMS & PolyDiMethylSiloxane \\
\hline RPM & Rotation Per Minute \\
\hline RT & Room Temperature \\
\hline SB & Soft Bake \\
\hline $\mathrm{SbF}_{6-}$ & Triarylsulfonium Hexafluoroantimonate \\
\hline SEM & Scanning Electronic Microscopy \\
\hline STA & Streptavidin \\
\hline TPA & Two Photon Absorption \\
\hline TPP & Two Photon Polymerization \\
\hline UV & Ultra Violet \\
\hline
\end{tabular}




\section{Introduction}

\subsection{Microtechnology}

The art and science of fabricating structures with nano-/micrometric dimensions as well as precision is of the immense concern to anyone investigating into nano-/microtechnology. The combination of micro, nanoscience and engineering is extremely relevant to biological applications because key building blocks of the biological systems fall into micro- and nanoscales: from small tissues, to single cells $(\sim 10 \mu \mathrm{m})$, to organelles $(100 \mathrm{~nm}-1 \mu \mathrm{m})$, and to proteins, nucleic acids, and other macromolecules (1-100 nm).

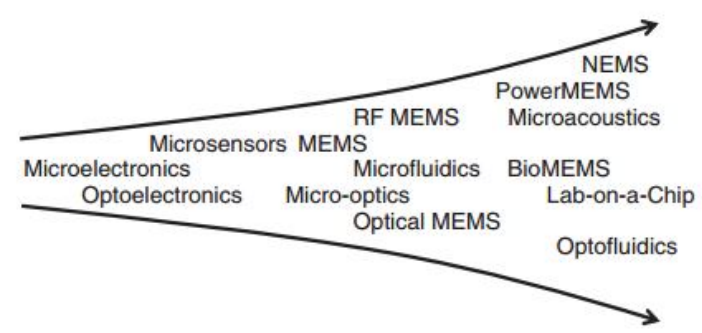

Figure 1. Evolution of microtechnology subfields from the 1960 onwards.

Microtechnology focuses on creating new technologies that combine inorganic materials (silicon chips, optical fibers, etc.) with organic materials (polymers, cells, etc.) at the micrometer-scale. Among all the aspects of microtechnology, fabrication techniques are one of the most important. In the mid-1990's, MEMS (Microelectromechanical systems) components began appearing in commercial products and applications including accelerometers used to control airbag deployment in vehicles, pressure sensors for medical applications, and inkjet printer heads. Today, MEMS devices are also found in projection displays or in data storage systems. Fig. 1 shows the evolution of the microtechnology since 1960s. The interdisciplinary nature of MEMS utilizes engineering and manufacturing expertise from technical areas including integrated circuit fabrication, mechanical, electrical, chemical engineering, as well as fluid engineering (microfluidics), optics, instrumentation and packaging. The emerging MEMS industry is already a multi-billion dollar market. It is expected to grow rapidly and become one of the major industries of the 21 st century.

Bio-MEMS systems focus on mechanical parts and microfabrication technologies made suitable for biological applications. Fig. 2 shows an example of a microfluidic array with cellular valving for single cell co-culture made from replica moulding in poly(dimethylsiloxane) (PDMS). 
Another recent example for Bio-MEMS is a two-component 3D polymer scaffold with distinct mechanical and protein-binding properties made by direct laser writing. Microfluidics has been used to perform a variety of biological assays (Khandurina et al. 2002; Schulte et al. 2002) with the two main advantages: minimal reagent consumption and parallelization. In particular, the dimensions $(10-100 \mu \mathrm{m})$ of microfluidics have made assays of live cells a popular application.

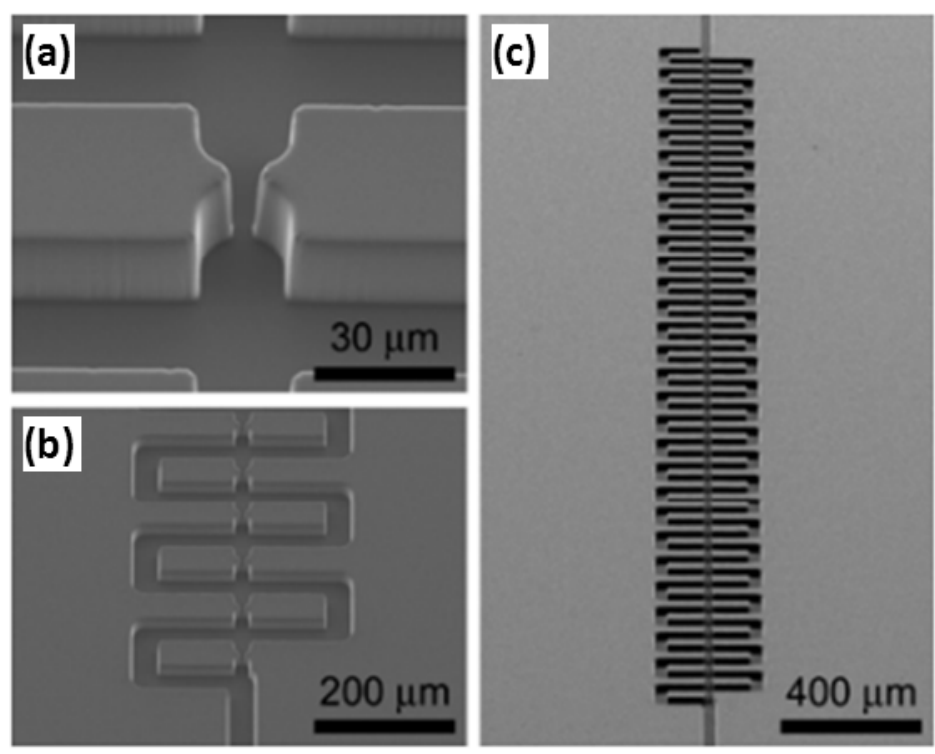

Figure 2. Microfluidic array with cellular valving for single cell co-culture. A single microstructured cell trap (a), a series of 6 traps (b) and an entire microfluidic circuit (c).(Frimat et al. 2011)

\subsection{Microfabrication}

Microfabrication is a group of processes used to construct physical objects with dimensions in the micrometer to millimeter range with its roots in the fabrication methods developed for the semiconductor industry driven by the demand to produce smaller features and more complex functional structures (Campbell 2001; Jaeger 2002; Plummer et al. 2000). In a microfabrication process, one takes a substrate and builds a device either out of its bulk material (bulk micromachining (Kovacs et al. 1998) or on its surface (surface micromachining (Bustillo et al. 1998). Often, the two are combined. In either case, four basic processes are used. One is lithography, which transfers a pattern into a material by irradiation with a beam (light, electrons, etc.). The second is growth or deposition of a thin-film (usually on the order of micrometers in thickness) onto a substrate. Etching creates features by selectively removing materials (either thin films or substrate) in defined patterns. The fourth type of process is bonding, where two substrates (often structured and with thin films) are bonded together. (Madou 1997; Ristic 1994). 
Lithography by polymerization of photosensitive materials using spatially selective light exposure is an active research field that utilizes the relatively easily achievable electromagnetic radiation as a source. Direct laser writing is already suitable for micro-structural patterning but employing non-linear interaction can help improve pattern resolution into sub-micrometer scale.

\subsubsection{Two photon polymerization}

In the second half of the 1990's two-photon polymerization (TPP) a laser-based nonlinear technique was introduced as a three-dimensional (3D) fabrication method to produce structures in the sub-micrometer regime (Maruo et al. 1997). Since then, TPP has attracted attention in fields like photonics, electronics, telecommunication and biomedicine for applications such as MEMS, information storage, plasmonics, metamaterials and tissue scaffolding (Kelemen et al. 2006; Rodrigo et al. 2009; Ovsianikov et al. 2007; Gittard et al. 2010). TPP is a direct laser writing technique, which means that moving the focused laser beam in three-dimension inside the material results in the fabrication of freestanding, readily assembled, fully 3D structures. (Malinauskas et al. 2013).The method has been extensively characterized in terms of achievable feature size (Malinauskas et al. 2013), polymer materials (Ostendorf et al. 2006) etc.

TPP requires four basic components: an ultrafast laser as light source, a high numerical aperture (NA) objective to focus it into a diffraction-limited spot, a photopolymer that has negligible linear absorption at the wavelength of the laser and a 3D positioner system that can translate the focal spot within the photopolymer with nanometer precision. The wavelength of the available lasers is typically that of the ultrafast-pulsed Ti:sapphire laser $(\sim 780 \mathrm{~nm})$ that can also be achieved with the recently-developed doped fiber laser systems. This value matches the double of the wavelength where most of the photopolymers have high linear absorption $(350-400 \mathrm{~nm})$. When the beam of such a laser (typical repetition rate: $100 \mathrm{MHz}$, pulse energy: 10-100 pJ, pulse duration: $100 \mathrm{fs}$ ) is tightly focused by high NA objective into the volume of the photosensitive material, the polymerization process can be initiated by non-linear absorption within the focal volume (Malinauskas et al. 2013). If I is the intensity of light, the resulting material photomodification scales as $\propto \mathrm{I}^{\mathrm{N}}$ with $\mathrm{N}>1$ being the order of nonlinearity. This means that instead of one, 2 or 3 photons are absorbed simultaneously by the material.

TPP has been implemented with a variety of acrylate, epoxy, and organic-inorganic hybrid materials. The common components of all this is the initiator that usually acts as the chromophore (absorbs the photon of the laser beam) and the monomer that crosslinks as the result of a 
photochemical process. We can distinguish radical-based and ionic polymerization, depending on the product of the initiator-photon interaction. The main steps of a cationic polymerization process are the following: initiator protonation resulted by photon absorption (IN $+\mathrm{h} v \rightarrow \mathrm{IN}-\mathrm{H})$, monomer activation $(\mathrm{MO}+\mathrm{IN}-\mathrm{H} \rightarrow \mathrm{MO}-\mathrm{H}+\mathrm{IN})$ and cross linking $(\mathrm{MO}-\mathrm{H}+\mathrm{MO} \rightarrow \mathrm{MO}-\mathrm{MO}-$ $\mathrm{H}$ ). Here IN is the initiator (IN-H: protonated initiator), hv is the energy of the absorbed photon, $\mathrm{MO}$ is the monomer. In a single photon absorption process, an initiator only absorbs one UV photon, while in a two-photon absorption (TPA) process the initiator absorbs two near infrared (NIR) photons simultaneously (Fig. 4(b)). Since the cross section for two photon absorption is orders of magnitude lower than for single photon absorption, the excitation beam intensities should be in order of $\mathrm{TW} / \mathrm{cm}^{2}$. At this high photon density two-photon absorption occurs at sufficiently high probability within the focal volume to generate sufficiently high number of activated initiator to start the polymerization. At NIR wavelengths the used photosensitive resins are transparent therefore the polymerizing beam can be focused into and translated in 3D within the volume of the resin without loss, as illustrated in Fig. 4(d) (Shuhui et al. 2006).
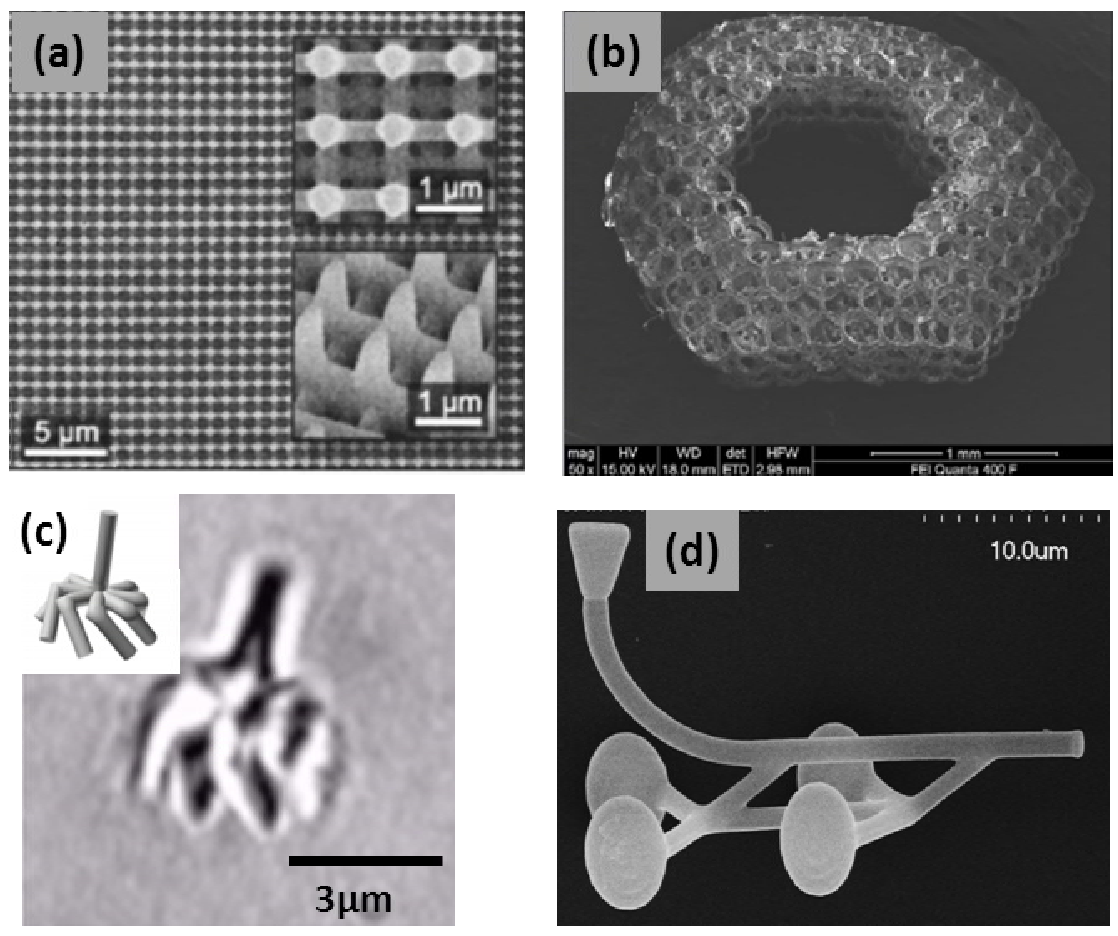

Figure 3. SEM micrographs of 2PP fabricated microstructures. (a) 3D photonic crystal structure, (b) cell scaffold from (PEGDA), (c) conical propeller and (d) a bent waveguide with spheroidal handles for optical trapping and actuation. 
Effectively,TPP is constrained to a small volumetric pixel called voxel. The size of the voxel is determined by optical parameters (focal spot size), chemical effects (the region where enough initiator is activated) and by viscosity (diffusion of the activated initiator) (Sun et al. 2003). The typical reproducible lateral feature size of a 3D structure produced by a single-beam TPP setup is around $200 \mathrm{~nm}$, while with various enhancement methods (Fischer et al. 2013) it can be reduced below $80 \mathrm{~nm}$. After crosslinking of the monomers along the illuminated trajectory, the unpolymerized resin is removed by a suitable solvent (development), leaving the polymerized solid structure either attached to the substrate or free-floating.TPP can produce a wide range of components or devices such as photonic crystals (Fig. 3(a)) (Deubel et al. 2006; Trull et al. 2011; Turner et al. 2011) or micromechanical (Fig. 3(c)) and microfluidic devices (Galajda et al. 2002; Schizas et al. 2010; Amato et al. 2012).Within the last couple of years biomedical applications were also addressed, especially within the fields of tissue engineering and cell culture scaffolds (Fig. 3(b)) to mimic in vivo 3D environments (Ovsianikov et al. 2010; Raimondi et al. 2012) or by studying single cell attachments and mechanics (Franziska et al. 2010). 3D microstructures produced by TPP have recently became available for applications in conjunction with optical tweezers (Fig. 3(d)) (Pollard et al. 2010; Rodrigo et al. 2007; Rodrigo et al. 2009; Palima et al. 2012) The combination of TPP structures and optical tweezers is yet to be used in biological experiments; however it is expected to be a sophisticated, sensitive and localized means to manipulate or probe various target objects (proteins, DNA, and single cells).

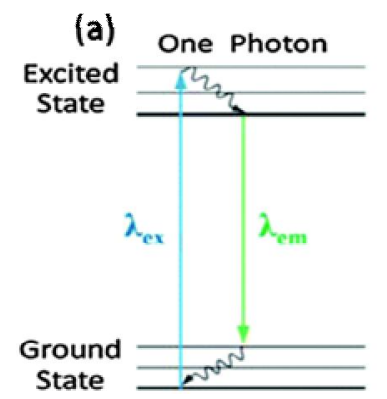

(b)

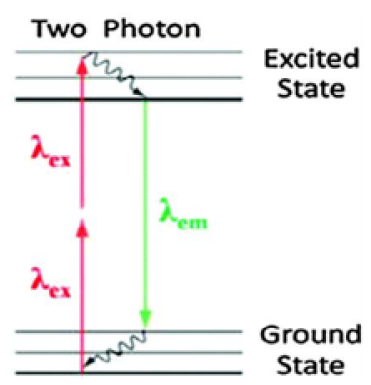

(c)

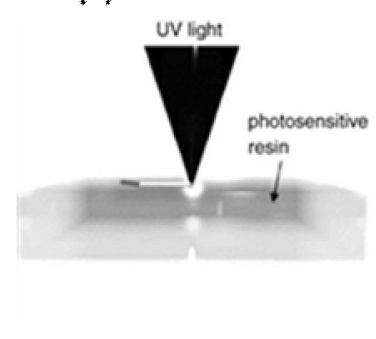

(d)

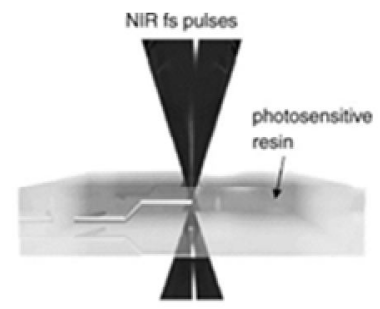

Figure 4. Simplified Jablonski diagrams for (a) one-photon absorption and (b) two photon absorption. Representation of the polymerization via single photon absorption (c) when the process takes place mostly on the photoresist surface. During two-photon polymerization (d) light can be focused into the volume of the photoresist and can be used for truly $3 \mathrm{D}$ structuring.

Polymerization of a complex microstructure with fine features can take up to several minutes, therefore TPP microfabrication in large quantities can be a time consuming process. A 
straightforward way to speed up the process is to redirect the original laser beam into multiple polymerizing foci, allowing the better utilization of the available laser power, which is usually much higher, than required for a single focus. This can be achieved by various diffractive optical elements, such as kinoforms (Kelemen et al. 2007), microlens arrays (Kato et al. 2005; Formanek et al. 2006) and spatial light modulators (SLM) (Gittard et al. 2011; Obata et al. 2010; Ritschdorff et al. 2012) modulating either the phase or the intensity of the laser beam. This ability of an SLM has been used to demonstrate parallel single-photon polymerization of multiple identical

microstructures by holographically created and translated focal spots (Jenness et al. 2008). Recently TPP of complex 3D microstructures was demonstrated where each individual microstructure was polymerized with the coordinated translation of multiple holographic foci Vizsnyiczai et al. (2014).

\subsection{Optical tweezer}

The optical tweezers effect is based on the idea that light carries momentum and when it is refracted by a small object, the change of momentum is transferred onto the object therefore a force acts upon it. The first modern day demonstration of this phenomenon came from Arthur Ashkin (Ashkin 1970). Optical trapping can typically be carried out on objects smaller than a couple of tens of micrometers, the index of refraction of which being higher than their surrounding media at the wavelength of the trapping laser. The typical forces that light can exert on these objects are lower than a few tens of $\mathrm{pN}$. Presently, there are wide ranges of implementation of the optical tweezer, the single beam version being the most widely used. Single beam optical trap is formed by tightly focusing a laser beam with a high NA microscope objective. Through optical trapping the movement of the target objects is confined in the immediate vicinity of the focal spot. The full theory of optical tweezers is out of the scope of this thesis.

There are two simplified models by which the force acting on the trapped object can be calculated (Neuman et al. 2004). When the trapped particle is much larger than the wavelength of the trapping laser, the conditions for Mie scattering are satisfied and the effect can be explained by ray optics. On Fig. 5 the trapped particle is displaced axially below the laser focus and incident rays ' $a$ ' and ' $b$ ' are focused to a point ' $f$ '. The rays that illuminate the particle of higher index of refraction are deflected by it and therefore their momentum is changed. According to Newton's third law, this momentum change of the photons imparts an equal and opposite momentum 
change to the particle which experiences a force that points toward the focal spot $\left(\mathrm{F}_{\text {total }}\right.$ : restoring force). A similar reasoning shows the emergence of a restoring force for any lateral displacement. This force, pointing towards the gradient of the focused beam, is also called gradient force. There's another force that acts on the particle: the scattering force is the result of the part of the beam that scatters off the object and pushes it in the direction of light propagation. For particles much smaller than the wavelength of the trapping laser, the conditions for Raleigh scattering are satisfied and they can be treated as dipoles that feel a Lorentz force due to the gradient in the electric field. For particle sizes in between, neither the Mie-scattering nor the dipole approach works, and more complex electromagnetic theories are required to describe the trapping force.

The optical trap typically interacts with biological objects either by directly trapping it or by trapping an intermediate manipulator object that is bound to it (classically a micrometer-sized transparent bead). The latter happens when the object is too small (DNA, protein) or sensitive (live cell) or has small refractive index contrast. The technique of indirect trapping has been widely used as a powerful manipulation and measurement technique in the biological and materials sciences (Mohammad et al. 2014) for instance in biophysics, where the mechanical behaviour of biological macromolecules can be investigated at the single molecule level (Forth et al. 2013; Moffitt et al. 2008; Hilario et al. 2010; Oroszi et al. 2006).

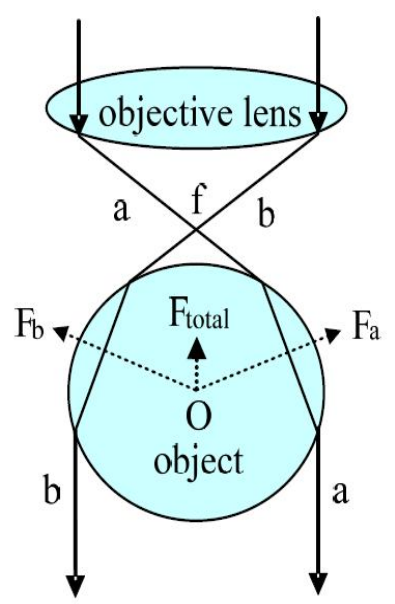

Figure 5. Ray-tracing explanation of the axial trapping force generation on a particle that is much larger than the wavelength of the trapping laser.

\subsubsection{Measuring forces with optical tweezers}

For small displacement the restoring force $\mathrm{F}$ is linearly dependent on the displacement $\mathrm{x}$ from the equilibrium position 


$$
\mathrm{F}=-\mathrm{kx}
$$

where $\mathrm{k}$ is the optical trap stiffness. Therefore to measure the force precisely, one has to measure the displacement precisely. Modern image analysis techniques are able to measure the position of a micron-sized bead with an accuracy of $2 \mathrm{~nm}$. Other techniques based on projecting the trapping beam or a measuring laser beam onto position sensitive diodes can also be used to measure the position of a bead more accurately. In case of any displacement the bead deflects the beam from its original position and the detected voltage signal reflects the amount of displacement (Williams 2002).

\subsubsection{Force calibration}

In order to determine the force, the instrument must be calibrated i.e to obtain the constant $\mathrm{k}$ (Neuman et al. 2004). One way to do this is the viscous drag method. If a liquid with viscosity $\eta$ flows past a sphere of radius $r$ with velocity $v$, the force due to viscous drag $F_{v i s}$ is given by:

$$
F_{v i s}=6 \pi \eta r v
$$

$\mathrm{F}_{\mathrm{vis}}$ can be calculated and if the bead is in equilibrium at a measurable distance $\mathrm{x}$ relative to its original position, this force will be equal to restoring force $\mathrm{F}=-\mathrm{kx}$, from where $\mathrm{k}$ can be calculated. Another method relies on that every trapped object is subject to thermal fluctuations. The trap stiffness can then be determined with the Equipartition theorem where $\mathrm{k}$ is calculated by measuring the variance of displacement distribution $\mathrm{x}$ :

$$
1 / 2 \mathrm{k}_{\mathrm{B}} \mathrm{T}=1 / 2 \mathrm{k}<\mathrm{x}^{2}>
$$

where $\mathrm{k}_{\mathrm{B}}$ is Boltzmann's constant, $\mathrm{T}$ is the absolute temperature and $\left\langle\mathrm{x}^{2}\right\rangle$ is the variance. For the power spectrum method the distribution of displacements has to be Fourier-transformed first. The one-sided power spectrum for the trapped bead is then

$$
S_{x x}(f)=\frac{k_{E} T}{\pi^{2} \beta\left(f_{0}^{2}+f^{2}\right)},
$$

where $\mathrm{k}_{\mathrm{B}}$ is Boltzmann's constant, $\mathrm{T}$ the absolute temperature, $\beta$ is the hydrodynamic drag coefficient of the object (for a sphere of radius $r$ in a medium with viscosity $\eta$ : $\beta=6 \pi \eta r$ ), and $f_{0}$ is the roll off frequency, related to the trap stiffness $\mathrm{k}$ through $\mathrm{f}_{0}=\mathrm{k}(2 \pi \beta)^{-1}$. The power spectrum must be fit with $\mathrm{f}_{0}$ from what $\mathrm{k}$ is obtained.

\subsubsection{Holographic optical tweezers}

In past decade the method to create large numbers of high-quality optical traps in arbitrary threedimensional configurations has been developed (Liesener et al. 2000). One of the ways to achieve 
it is holographic optical tweezers (HOT) that uses a computer-controlled diffractive optical element (DOE) to split a single collimated laser beam into several separate beams, each of which is focused at the trapping focal point by a high NA objective (Dufresne et al. 1998; Dufresne et al. 2001). The DOE in these setups is a spatial light modulator (SLM) which realizes point by point phase modulation throughout the beam cross section by a liquid crystal layer. With SLM it is possible to create and dynamically rearrange multiple focal spots in 3D in real-time (Bianchi et al. 2010). The enhanced capabilities of such dynamic HOT systems (Fig. 6) offer new opportunities for research and engineering, as well as new applications in biotechnology, nanotechnology and manufacturing.

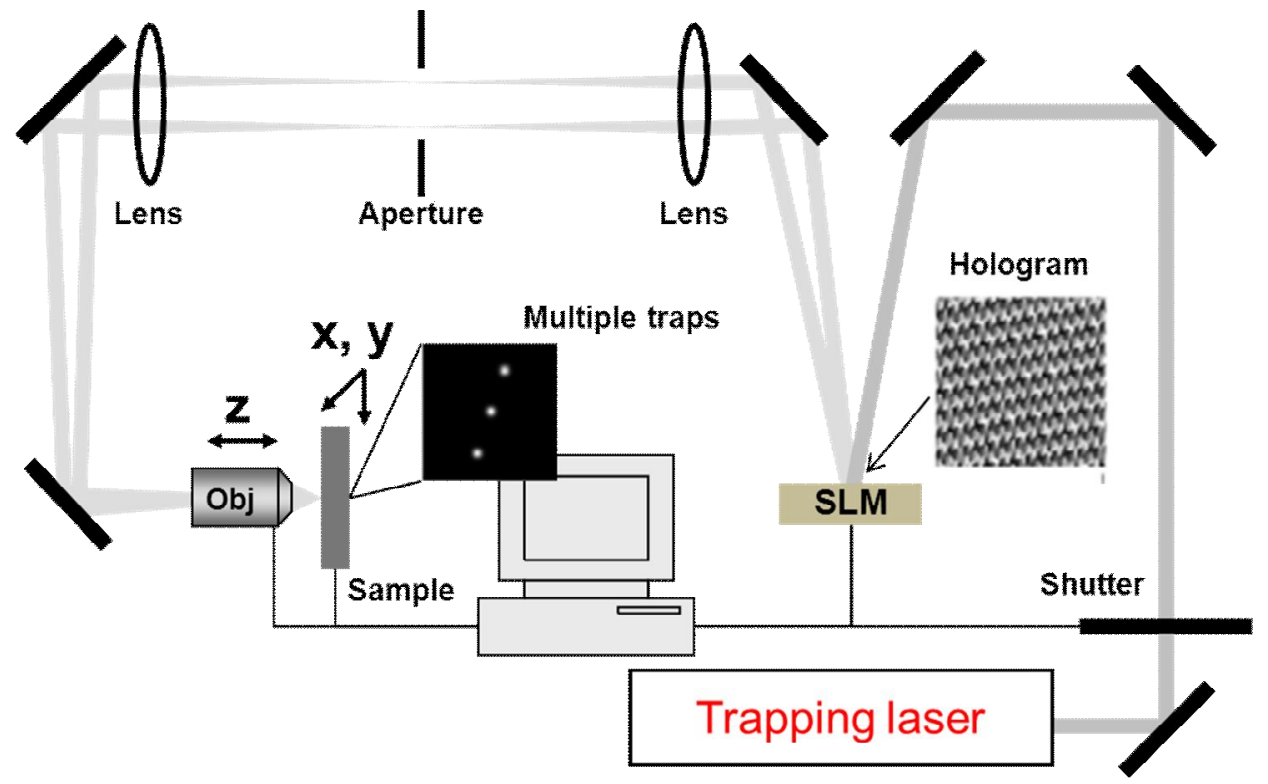

Figure 6. Schematics of a holographic optical tweezers using a reflective liquid crystal spatial light modulator (SLM).

\subsection{Photoresist SU-8}

\subsubsection{Chemical components}

SU-8 is an epoxy-based negative tone photoresist invented by IBM in 1989 (Gelorme et al. 1989); (LaBianca et al. 1995) made by dissolving EPON ${ }^{\circ}$ SU-8 resin (a registered trademark of Shell Chemical Company) in an organic solvent such as propylene glycol methyl ether acetate (PGMEA), cyclopentanone or gamma-butyrolactone (GBL) and adding up to $10 \mathrm{wt} \%$ of triarylsulfonium hexafluoroantimonate $\left(\mathrm{SbF}_{6^{-}}\right)$salt as a photoinitiator. The amount of solvent determines the viscosity and thus, the range of available thicknesses, extending from tens of nanometers to millimeters. A single monomer molecule contains eight epoxy groups hence the 
name SU-8 (Fig. 7(a)). The photoinitiator is sensitive to near-ultraviolet light (350-400 nm). SU-8 has been used for a number of applications such as microfluidic chambers and optical components including lasers, lenses and waveguides (El-Ali et al. 2004; Balslev et al. 2006; Wang et al. 2004). This is due to its good mechanical and thermal properties as well as the excellent chemical resistance and biocompatibility (Voskerian et al. 2003). Additionally SU-8 is highly resistant to many organic and inorganic solvents.

\subsubsection{Photopolymerization of SU-8}

(a)

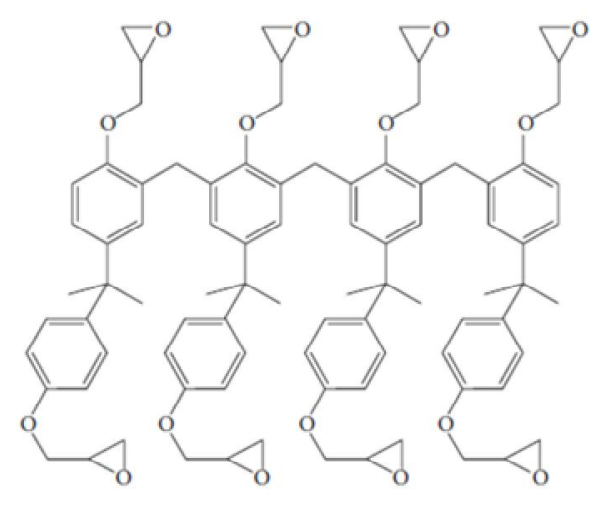

(b)
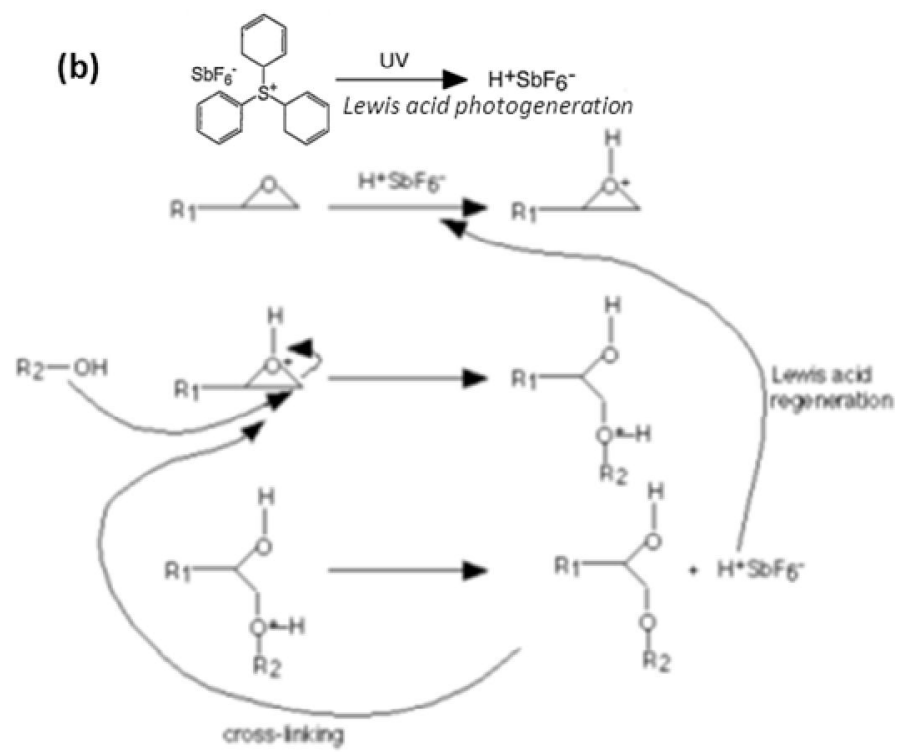

Figure 7. (a) Chemical structure of the SU-8 monomer with eight epoxy-groups (Shaw et al. 1997)

(b) Steps of the chemical process leading to the cationic polymerization of SU-8.

SU-8 polymerizes by cationic polymerization. The actual mechanism of polymerization is quite complex (Crivello 1999). The simplified mechanism is represented at Fig. 7(b). During the step of photoactivation the initiator $\left(\mathrm{SbF}_{6}\right)$ is protonated due to the UV-radiation and a strong acid is formed (Lewis acid). The generated catalytic protons have an affinity to the oxygen of the epoxygroups on the SU-8 monomer, which are partially negatively charged due to the free electron pairs (Genolet 2001). The epoxy-ring is opened with the reaction of another monomer (cross linking). The new formed group has an extra proton which regenerates the Lewis acid which is again available to activate another epoxy group. The cross-linking is continued and leads to the formation of polymer clusters and finally to a gel state. Termination of the propagating chains can 
occur by reaction with anions, solvent molecules or other species present in the reaction system (George 1981).

\subsubsection{SU-8 processing}

Fig. 8 is an overview of the different steps involved in the processing of SU-8. For photolithography applications first a layer of SU-8 must be created. This is done by spin coating, where the layer thickness is determined by the rotation speed and the amount of solvent mentioned before.

(a)

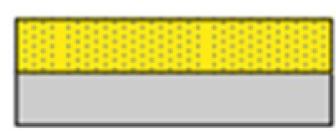

(b)

Silicon

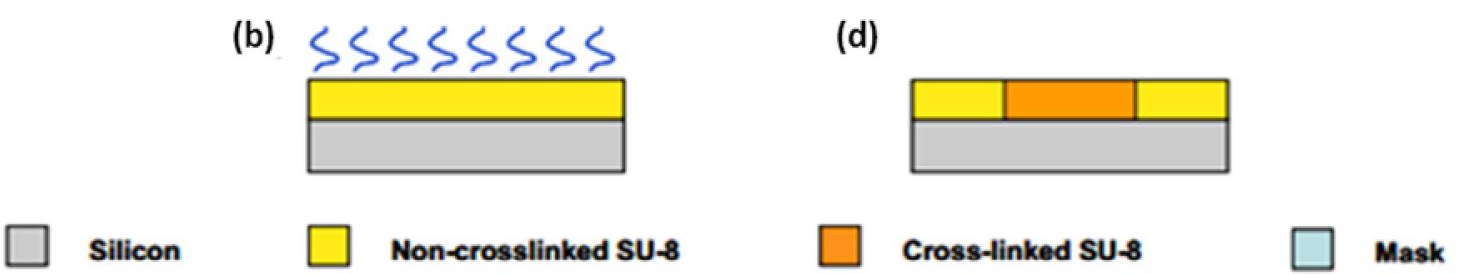

(c)

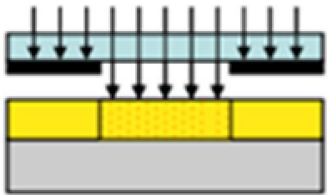

(d) (e)

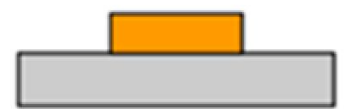

Figure 8. Processing steps of SU-8 structures: (a) spin-coating, (b) soft-bake, (c) exposure, (d) post exposure-bake and (e) development (Keller 2008).

The goal of the second step (soft-bake, SB) is to reduce the amount of solvent as much as possible in the photoresist layer, thereby increasing its viscosity. Conventionally, SB is done on a hotplate. The exposure step is responsible for the photo-initiation, usually done with UVradiation (e.g. $\lambda=365 \mathrm{~nm}$ ). The exposure dose determines the concentration of photo-acid that is released and that contributes to the polymerization. The actual polymerization is taking place during post exposure bake (PEB) which is usually done immediately after exposure to limit photoinitiator diffusion into non-exposed areas. For development (removal of unpolymerized resin) of the SU-8 patterns, the substrate is immersed in an appropriate solvent (e.g. Propylene glycol methyl ether acetate or PGMEA). The development time is dependent on the layer thickness. After the development, the substrate is rinsed with alcohol and dried.

\subsubsection{SU-8 surface modification:}

Polymeric materials of different chemical characteristics, such as ORMOCER, SU-8, S1813, and PEGDA have been used to create 2D and 3D micro and nanostructures of arbitrary complexity 
(Kelemen et al. 2006; Ovsianikov et al. 2007; Gittard et al. 2010). Many applications, especially in sensory or tissue engineering, require the functionalization of the microstructure's surface. Depending on the goal, the coating can consist of small functional groups, larger biomolecules (protein, DNA) or even metal nanoparticles. The coating strategy depends primarily on the material of the photoresist used to make the microstructures: in our case its SU-8 (Tao et al. 2006; Chen et al. 2007).

Bare surface of cross linked SU-8 does not easily allow covalent immobilization of biomolecules directly therefore it should be modified so as to have at least one functional group, i.e. amine, thiol, aldehyde, carboxyl, etc., for anchoring biomolecules by stable covalent bonds. The existing coating strategies often take advantage of the opening of the epoxide groups (Tao et al. 2006). Ceric ammonium nitrate (CAN) has long been known and used to catalyze epoxy ring opening (Iranpoor et al. 1995; Wang et al. 2007). CAN treatment changes the protein binding capacity of SU-8 as it was determined for Immunoglobulin G (IgG) (Blagoi et al. 2008). Using longer bisamino-terminated n-alkane linkers SU-8 micro-actuators were coated with gold nanoparticles to seed a conducting $\mathrm{Cu}$ layer (Dai et al. 2008). In this work I used amine group creating techniques, as introduced below:

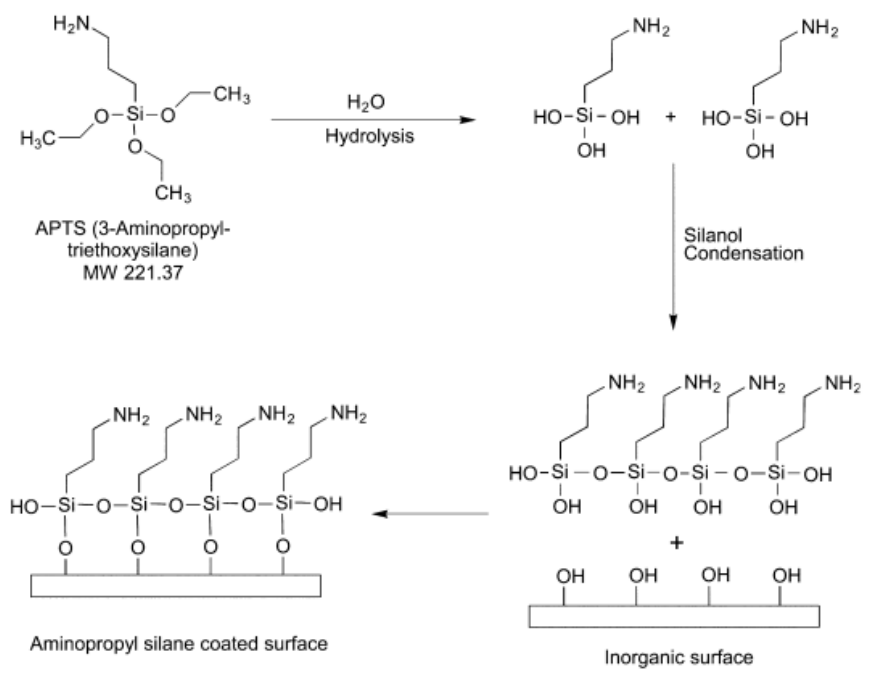

Figure 9. The deposition of APTES onto a surface containing hydroxyl groups results in the formation of a covalent coating containing primary amine groups. 


\subsubsection{Silanization}

Silanization is the covering of a surface through self-assembly with organofunctional alkoxysilane molecules. Glass or metal oxide containing surfaces can be easily silanized, because they contain hydroxyl or silanol groups which are taking part in forming covalent -Si-O-Si- bond. The goal of silanization is to create surface functional groups as anchors for further surface modifications. Reaction can be done in aqueous solution (Fig. 9), in organic solution and even in the vapor phase. The first step in the reaction involves the hydrolysis of the alkoxysilane groups to form highly reactive silanols. The silanols undergo hydrogen bonding with other silanols in solution and on the substrate, resulting in a network of associated organosilane derivatives. A condensation reaction then takes place to form a polymerized coating of the organosilane on the surface of the substrate.

\subsubsection{PEGylation:}

PEGylation is the process of attachment homo or hetero bifunctional polyethylene glycol (PEG) polymer chains to molecules or modified surfaces. The choice of the suitable terminal functional group for the PEG derivative (e.g. thiol, amine, and carboxyl) is based on the type of available reactive group on the molecule to which the PEG will be coupled. For instance for proteins, the reactive groups are amino acids. Poly or oligo(ethylene glycol)-grafted surfaces are widely used as passivation layers to avoid nonspecific adsorption of proteins (McPherson et al. 1998; Delamarche et al. 2003; Papra et al. 2001) or as immobilization matrixes for peptide microarrays (Houseman et al. 2002) and biosensors (Ruiz-Taylor et al. 2001; Stadler et al. 2004).

\subsection{Fluorescence enhancement: the phenomenon}

Fluorescence spectroscopy is entrenched throughout the life sciences today and is used for a variety of applications, including the detection of chemical or biological analytes/species, in reporting molecular events or for the understanding of microenvironments, such as local viscosity, polarity and voltage measurements, to name but just a few. In the majority of these fluorescence based measurements, fluorophore brightness and photostability are the primary concerns and limitations to the fluorescence technique, with little opportunity existing to tune favorably the photophysical properties of the fluorophore (Geddes 2013). It was found,however, that many kinds of metal NPs can dramatically enhance or quench the fluorescence of fluorophores along with the changing of separation distance between them. Such fluorescence enhancement is known as metal enhanced fluorescence (MEF). Noble metal nanoparticles (NPs) 
have been widely applied for MEF in chemical and biological sensing (Anker et al. 2008; Mayer et al. 2008; Haes et al. 2002; Raschke et al. 2003), or bioimaging technologies (Durr et al. 2007; El-Sayed et al. 2005) due to their unique shape- and size-dependent localized surface plasmon resonance (LSPR). Furthermore, when the wavelength of fluorescence emission overlap well with the plasmonic response of metal NPs, the fluorophores can couple with metal NPs, which result in a shortening of lifetime and an increasing of photostability as compared with that of free fluorophores (Zhang et al. 2007; Amiot et al. 2008). Improvement of sensitivity and spatial resolution promises new possibilities in identification and characterization of specific biomolecules (Goldys 2009). MEF achieved by NPs has been applied recently in cell imaging observing otherwise weakly detectable signals (Matveeva et al. 2007; Gartia et al. 2011; Hong et al. 2011); movable enhancers in the range of 100 um were shown to induce fluorescence enhancement of nearly a factor of 10 in cell imaging as determined with confocal microscopy by Radha et al. (Radha et al. 2010); micromirrors were also generated of gold NPs by Kim and Osterloh with possible enhancement application (Kim et al. 2006).

\subsubsection{Principle of metal fluorescence enhancement}

The demonstrations and applications of MEF are a decade old, the theory of fluorescence enhancement with metallic surfaces and particles has developed since the 1980s. The photophysical properties of a fluorophore near the metal surface can be interpreted by a modified Jablonski diagram (Lakowicz 2001) (Fig. 10). The enhancement can be understood in terms of the fluorescence quantum yield $\left(\mathrm{Q}_{0}\right)$ and lifetime $\left(\tau_{0}\right)$. These quanta, in the free-space emission, can be given by $\mathrm{Q}_{0}=\Gamma /\left(\Gamma+\mathrm{k}_{\mathrm{nr}}\right)$ and $\tau_{0}=\left(\Gamma+\mathrm{k}_{\mathrm{nr}}\right)^{-1}$, where $\Gamma$ is the intrinsic radiative rate. In traditional fluorescence experiments, the change in quantum yield or lifetime is due to the change in the nonradiative decay rate $\left(\mathrm{k}_{\mathrm{nr}}\right)$, which results from the change in environment of the fluorophore, from quenching or from FRET.

A fluorophore can be pictured as an oscillating dipole (a radiating antenna). Nearby metal surfaces can respond to this oscillation and modify the rate of its emission. In order to calculate the quantum yield and lifetime for the fluorophores near metal particles or surfaces a new radiative decay rate term can be introduced $\Gamma_{\mathrm{m}}$ that modifies these values as shown:

$$
\begin{gathered}
\mathrm{Q}_{\mathrm{m}}=\left(\Gamma+\Gamma_{\mathrm{m}}\right) /\left(\Gamma+\Gamma_{\mathrm{m}}+\mathrm{k}_{\mathrm{nr}}\right) \\
\tau_{\mathrm{m}}=\left(\Gamma+\Gamma_{\mathrm{m}}+\mathrm{k}_{\mathrm{nr}}\right)^{-1}
\end{gathered}
$$


(a)
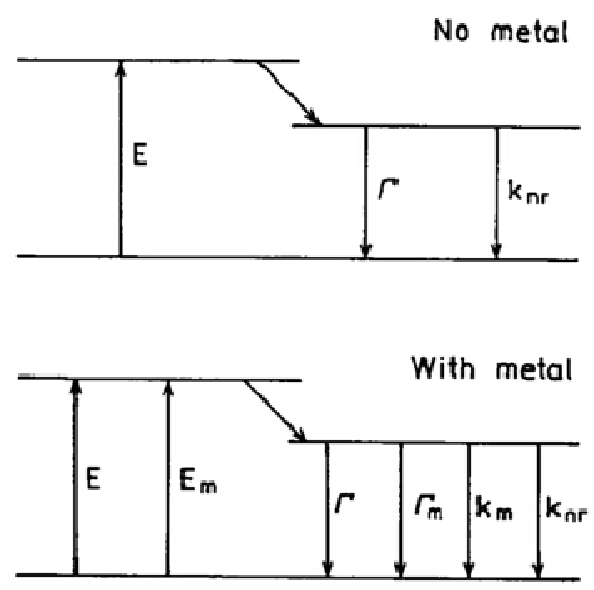

(b)

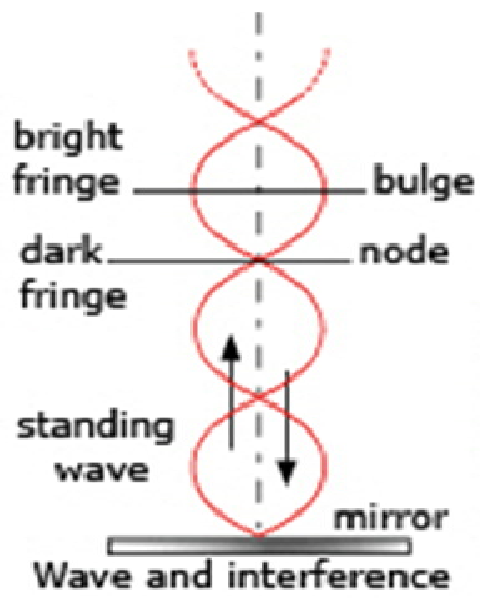

Figure 10. (a) Jablonski diagram of fluorescence for the free space condition and its modified form in the presence of metal. (b) The reflection of a light field in a mirror generates a standing wave. (http://www.nobelprize.org/nobel_prizes/themes/physics/biedermann/).

The incident excitation light also interacts with the metal and the modified field affects the fluorophore. These effects result in an increased detectability and often photostability.

Fluorescence enhancement can be achieved by setting the emitter close to a planar reflective (dielectric or metallic) surface ('mirror effect' (Drexhage 1970)). The principle of fluorescence enhancement on such substrates is based on their ability to reflect the light illuminating the sample and the light emitted by fluorophores. The interference between incoming and reflected electromagnetic fields leads to the formation of a standing wave close to the surface. Consequently, the excitation field intensity oscillates as a function of the distance from the mirror, alternating in-phase positions where the fluorophore excitation rate benefits from a maximum fourfold enhancement (antinode) and out-of-phase positions where the excitation rate decreases to zero (node) (Fig. 10(b)). In addition, the emission rate also changes, depending whether the reflected emitted light is in or out of phase with that just being emitted (Le Moal et al. 2007). 


\section{Aim of the thesis}

The objective of my work is to enable the use of two-photon polymerized 3D microstructures made of the photoresist SU-8 in biological applications such as fluorescence enhancement or indirect cell manipulation by functionalizing their surface. To fulfil this objective we proposed the following aims:

a) Effective silane-mediated functionalization of TPP 3D microstructures with the protein streptavidin and with gold nanoparticles.

- Characterization the effects of the different process parameters on the quality, integrity and surface morphology of the SU-8 structures through contact angle measurements, FTIR, AFM, SEM and optical microscopy.

- Implementation of the efficient biotin-streptavidin linkage on the surface of the microtools by covalently binding biotin to the SU-8 surface. This can further be used to link other biological objects to the microtools.

- Coating the surface of the polymer microtools with gold nanoparticles while controlling the NP density. This may enable their use in optical trapping experiment while still having enough nanoparticle density for surface-enhanced spectroscopic measurements.

b) Fluorescence enhancement by gold nanoparticle-functionalized, TPP 3D microstructures.

- Determination of the degree of localization of the fluorescence enhancement that can be achieved by the coated TPP microstructures.

- Resolving the effect of NP densities on the enhancement factor.

- Demonstration of the enhancement in that general case when the plane of the fluorescent layer is not perpendicular to the direction of the optical axis.

- Evaluation of the enhancement at different excitation wavelengths.

- $\quad$ Exploring the origin of the enhancement.

c) Indirect optical manipulation of single live cells by protein-coated TPP 3D microstructures.

- $\quad$ Testing different PEG-diamine based functionalization methods to realize the highest streptavidin density on the 3D microtools.

- $\quad$ Binding live cells effectively to complex 3D microtools taking advantage of the high-affinity biotin-streptavidin binding. 


\section{Materials and Method}

In this work I present functionalization methods for two-photon polymerized microstructures with sub-micrometer features and use the resulted structures for enhanced fluorescence observation and indirect optical cell micromanipulation. In this section, first I introduce the sample preparation steps: the polymerization of SU-8 layers and microstructures, their functionalization with various proteins and gold NPs using silane and PEG-based linker molecules, cell functionalization and further their characterization. Then the experimental setups and procedures for the enhanced fluorescence measurements and the indirect optical cell manipulation are explained.

\subsection{Fabrication of SU-8 layer by UV lithography}

The steps of the functionalization protocol were tested on large area SU-8 layers made by UV lithography on glass cover slips or on $\mathrm{BaF}_{2}$ windows for the FTIR measurements. The SU-8 process parameters of these layers were selected such that the relative amount of the epoxide groups remaining after cross-linking were similar as in the TPP-made structures. 2-3 $\mu \mathrm{m} \mathrm{SU-8}$ layers were made by spin coating (P-6708 spin coater, Specialty Coating Systems, USA) SU-8 2002 (Microresist GmbH, Germany) onto the substrates. The SU-8 layers were first soft-baked $\left(96^{\circ} \mathrm{C}, 2 \mathrm{~min}\right)$, illuminated with a UV photolithography flood exposure source (365 $\mathrm{nm} \mathrm{Hg}$ line, doses from $170 \pm 15$ to $16320 \pm 1440 \mathrm{~mJ} / \mathrm{cm}^{2}$, mode197435, Newport, Irvine, Ca, USA) then post-exposure baked $\left(96^{\circ} \mathrm{C}, 10 \mathrm{~min}\right)$ and developed (mrDev 600, Microresist $\mathrm{GmbH}$, Germany).

\subsection{Fabrication of 3D microstructures by TPP}

The two-photon polymerization of the fluorescence enhancers were carried out by a system built around a Zeiss Axiovert 40 CFL microscope (Palima et al. 2012; Kelemen et al. 2007). The setup is practically identical to that shown in Fig. 6 except the laser source is different. The ultra-short laser beam (C-Fiber A 780, Menlo Systems GmbH, Germany, Ds $=100$ fs, $\mathrm{k}=785$ nm, $100 \mathrm{MHz}$ repetition rate) was focused into an $\sim 18 \mu \mathrm{m}$ thick layer of soft-baked SU-8 2007 photoresist layer (spin coated with 700 RPM) by a $100 \mathrm{X}$ oil immersion (Zeiss Achroplan, NA $=1.25$ ) or by a $63 \mathrm{X}$ long working distance objective (Zeiss LD Plan-Neofluar, NA $=0.75$ ). In some cases we polymerized with multiple beams split by SLM (Pluto NIR, Holoeye, Germany) (Vizsnyiczai et al. 2014). During illumination the sample was moved relative to the focus by a 3D piezo translation system. (P-731.8L in X-Y and P-721.10 in Z, Physik Instrumente, Germany). Then the 
sample was baked and developed. The used laser power was between $2 \mathrm{~mW}$ and $10 \mathrm{~mW}$ at the back aperture of the objective and the scan speed was between $3 \mu \mathrm{m} / \mathrm{s}$ and $12 \mu \mathrm{m} / \mathrm{s}$ depending on the desired structure.

For developing the protein and NP fucntionalization methods we prepared TPP microstructures in the forms of blocks $(15 \times 15 \times 6 \mu \mathrm{m})$ (Fig. 11(c)), microspirals (radius 0.8-1 1 m, height $6 \mu \mathrm{m}$ ) (Fig. 11(b)) and optical tweezers microtools (OT-tools) (Fig. 11(a)). For the fluorescent enhancement studies (Fig. 11(d)) flat enhancer structures with parallel (E1) and with rounded sides (E2) were made as well as two other types with two (E3) or four tips (E4). The largest dimensions of each structure measured $15 \mu \mathrm{m}$ and $20 \mu \mathrm{m}$ while their thickness varied between 3 $\mu \mathrm{m}$ and $5 \mu \mathrm{m}$. The tips on E3-E4 structures were of 3-8 $\mu \mathrm{m}$ length and $\sim 1 \mu \mathrm{m}$ width. Additionally, flat (type F), substrate-attached blocks with 30 x $30 \mu \mathrm{m}$ lateral dimensions were also polymerized for micro-spectroscopic measurements. For the cell binding experiments ellipsoids with $6 \mu \mathrm{m}$ large axis and $4 \mu \mathrm{m}$ small axis and crosses with $5 \mu \mathrm{m}$ arms were polymerized (Fig. 11(b)) together with OT-tools similar to that on (Fig. 11(a)) except with a flat end.

(a)

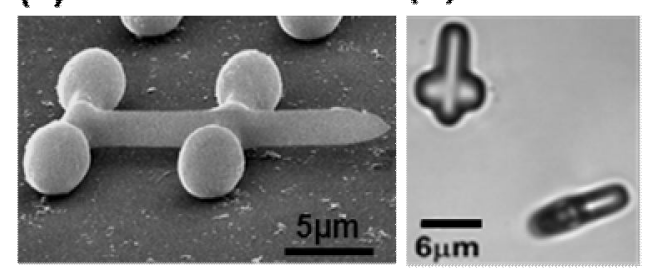

(c)

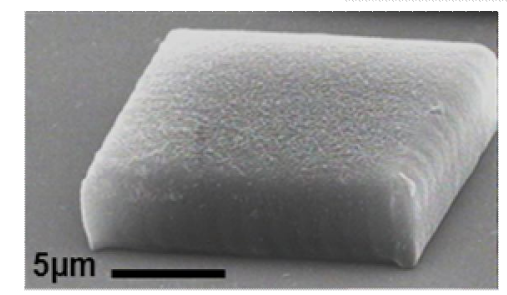

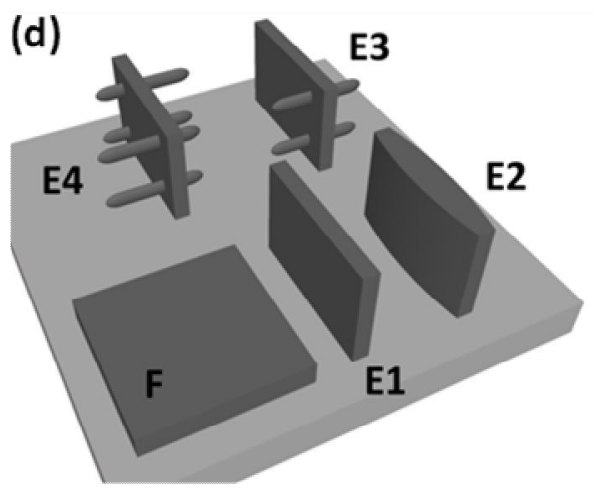

Figure 11. (a) Electron microscopic image of a two-photon polymerized optical tweezer microtool and (c) type F block, (b) optical microscopic image of TPP cross and (d) schematic drawing of the four types of enhancer structure (E1-E4) and the one used for micro-spectroscopy (F).

Our goal was to bind proteins specifically (using biotin) and nonspecifically (with glutaraldehyde) to the SU-8 structures. The process starts with creating $-\mathrm{OH}$ groups on the surface by epoxy ring opening, followed by introducing primary amines ( $-\mathrm{NH}_{2}$ groups) and then by incubating it in a biotinylation agent or glutaraldehyde. 


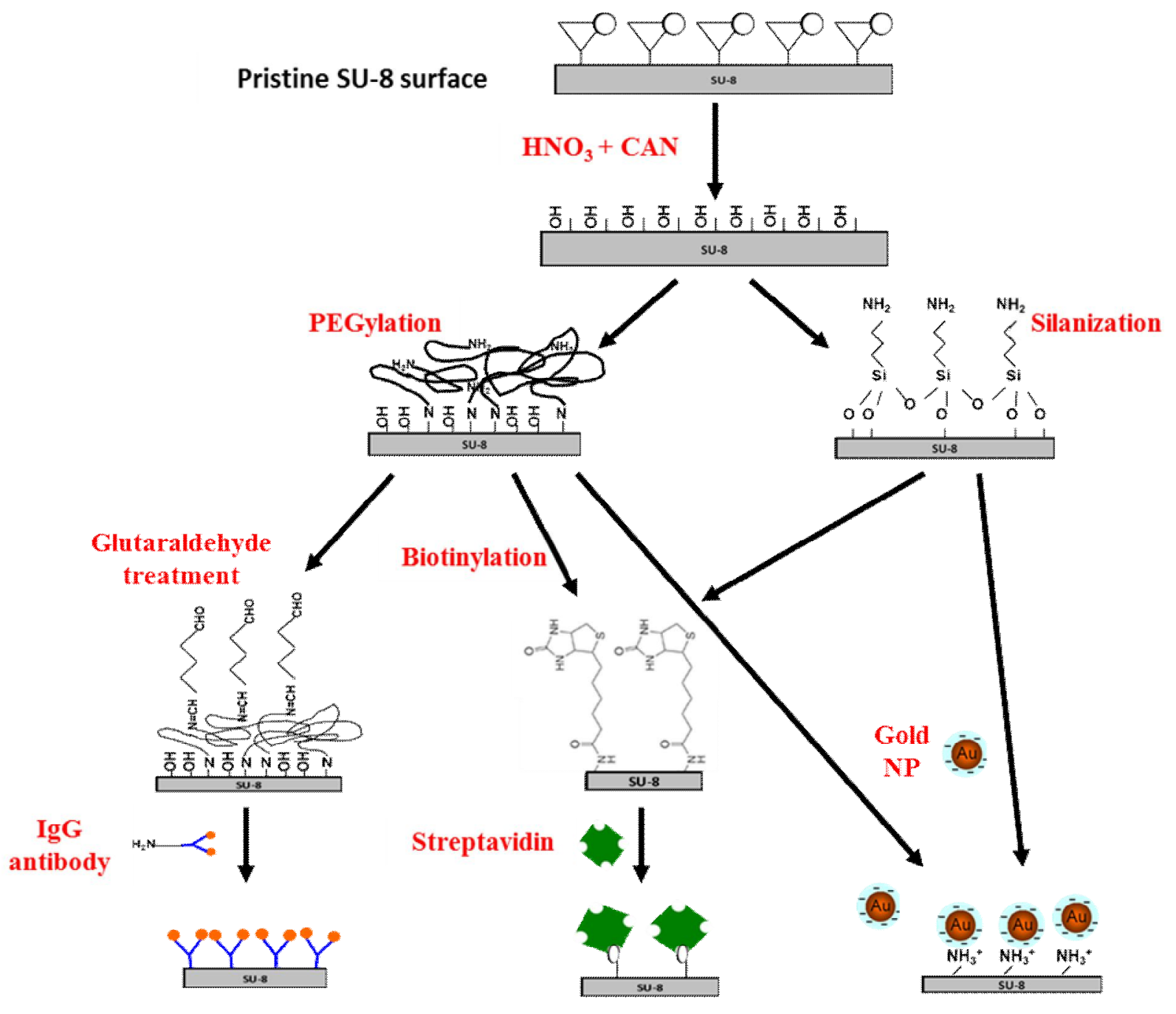

IgG antibody-coated SU-8

Streptavidin-coated SU-8

Gold NP-coated SU-8

Figure 12. Schemes of the different functionalization methods used in this work to modify the SU-8 surface.

The affinity of the surface toward protein was checked after each of these steps. Fig. 12 shows the schematics of different functionalization methods used in this thesis.

\subsection{Coating of SU-8 surfaces with protein}

\subsubsection{Coating via silanization}

The cured SU-8 structures were first incubated in the mixture of $1 \mathrm{M}$ nitric acid and $0.1 \mathrm{M} \mathrm{CAN}$ (Cerium (IV) ammonium nitrate, $\left(\mathrm{NH}_{4}\right)_{2} \mathrm{Ce}\left(\mathrm{NO}_{3}\right)_{6}$, Sigma-Aldrich) for $30 \mathrm{~min}, 2 \mathrm{hr}$ or $4 \mathrm{hr}$ at RT then rinsed with milliQ water and dried with a stream of nitrogen. The acid-treated samples were incubated in $2 \%$ APTES (3-Aminopropyl triethoxysilane, Sigma-Aldrich) dissolved in 
isopropanol for 90 min at RT to create free amine groups on their surface. Afterwards the samples were washed in isopropanol and in milliQ water for $10 \mathrm{~min}$ and dried with nitrogen. Sulfo-NHS biotin (cat No. 21217, Thermo Fisher Scientific Inc., USA) linker, freshly dissolved to $1 \mathrm{mg} / \mathrm{mL}$ concentration in PBS ( $\mathrm{pH}$ 7.4) was reacted with the silanized SU-8 for $4 \mathrm{hr}$ at RT. The biotinincubated surface was washed with PBS and immediately used for streptavidin (STA) incubation. We used DyLight650 labeled STA to avoid the autofluorescence region of SU-8 (Marie et al. 2006; Blagoi et al. 2008). We incubated the biotinylated SU-8 in 10 or $100 \mathrm{nM}$ STA conjugate in PBS (pH 7.4) overnight at RT, washed with PBS and finally with $1 \%$ BSA in PBS. Altogether, five types of surfaces were tested as detailed in Table 1.

\begin{tabular}{llll}
\hline Method \# & CAN+ HNO $_{\mathbf{3}}$ & APTES & NHS-Biotin \\
\hline Mthd 0 & No & No & No \\
Mthd 1 & Yes & No & No \\
Mthd 2 & Yes & Yes & No \\
Mthd 3 & Yes & No & Yes \\
Mthd 4 & Yes & Yes & Yes \\
\hline
\end{tabular}

Table 1: Description of surface treatment methods applied on the SU-8 surfaces via silanization, after which the streptavidin binding was evaluated.

\subsubsection{Coating via PEGylation}

The process of PEGylation starts with an acid treatment similarly as silanization. Then $10 \mu \mathrm{L}$ of $15 \mathrm{mM}$ PEG-diamine (Sigma, $\mathrm{Mw}=2000$ ) solution in methanol was applied on the samples (Schlapak et al. 2006) and the solvent was allowed to evaporate slowly. Then the slides were incubated at RT for 20 mins, rinsed in milli Q water and dried. For specific protein coating freshly dissolved sulfo-NHS biotin ( $1 \mathrm{mg} / \mathrm{mL}$ in PBS, $\mathrm{pH} 7.4)$ was reacted with the PEGylated SU-8 for $4 \mathrm{hr}$ at RT. After washing the slides were incubated with $10 \mathrm{nM}$ or $100 \mathrm{nM}$ streptavidin in PBS, washed and kept in PBS. Alternatively for non-specific functionalization, glutaraldehyde (2.5\% in PBS) was used to link the protein to the PEG-diamine coated SU-8. After rinsing with milliQ water two kinds of protein were used: fluorescently conjugated STA (10 nM) and an antibody $(7 \mathrm{nM})$ (IgG, Alexa 568 conjugated goat, anti-rabbit). Altogether, seven types of surfaces were tested as detailed in Table 2. 


\begin{tabular}{lllllll}
\hline Method \# & $\begin{array}{l}\text { CAN } \\
\text { + HNO }\end{array}$ & $\begin{array}{l}\text { PEG- } \\
\text { diamine }\end{array}$ & $\begin{array}{l}\text { NHS- } \\
\text { Biotin }\end{array}$ & $\begin{array}{l}\text { Glutar- } \\
\text { aldehyde }\end{array}$ & $\begin{array}{l}\text { Fluorescent } \\
\text { STA }\end{array}$ & $\begin{array}{l}\text { Florescent } \\
\text { Antibody }\end{array}$ \\
\hline Mthd 0 & No & No & No & No & Yes & No \\
Mthd 1 & No & Yes & No & No & Yes & No \\
Mthd 2 & Yes & Yes & No & No & Yes & No \\
Mthd 3 & No & Yes & Yes & No & Yes & No \\
Mthd 4 & Yes & Yes & Yes & No & Yes & No \\
Mthd 5 & Yes & Yes & No & Yes & Yes & No \\
Mthd 6 & No & No & No & No & No & Yes \\
Mthd 7 & Yes & Yes & No & Yes & No & Yes \\
\hline
\end{tabular}

Table 2: Description of surface treatment methods applied on the SU-8 surfaces via PEGylation, after which the streptavidin binding was evaluated. The highlighted Method 4 was used to functionalize the optical tweezers microtools.

\subsection{Preparation of a fluorescent protein layer on glass coverslip}

The procedure is similar to as described in section 3.2 until the APTES treatment then the glass slides were cured at $120^{\circ} \mathrm{C}$ for 10 mins. Finally the glass slides were incubated with a droplet of $\sim 100 \mu \mathrm{L}$ of $1 \mu \mathrm{M}$ biotinylated BSA (bBSA) in PBS (pH 7.4) in a humid environment at $4^{\circ} \mathrm{C}$ for 2 hr. The coverslips were then washed in PBS and incubated in $10 \mathrm{nM}$ fluorescent STA (DyLight 650, exc/em: $652 \mathrm{~nm} / 672 \mathrm{~nm}$ and Cy3, exc/em: $550 \mathrm{~nm} / 570 \mathrm{~nm}$ ) in PBS overnight at RT. The samples were kept in PBS for further use. The absorption of the resulted DyLight 650-conjugated fSTA layer at $650 \mathrm{~nm}$ was $\sim 4 \mathrm{mOD}$.

\subsection{Functionalization of SU-8 with gold NPs}

Aqueous gold nanoparticles were synthesized using the standard Turkevich method (Turkevich et al. 1951). Shortly, $100 \mathrm{~mL}$ of $\mathrm{HAuCl}_{4}\left(10^{-2} \% \mathrm{~m} / \mathrm{m}\right)$ solution was brought to boiling with continuous stirring, 1\% tri-sodium citrate 2-hydrate was added, boiled for another $10 \mathrm{~min}$ and finally allowed to slowly cool to RT. The $\mathrm{pH}$ was adjusted to $\sim 5.5$ resulting in negatively charged gold NPs. The diameter of the obtained NP was $80 \mathrm{~nm}, 41 \mathrm{~nm}, 24 \mathrm{~nm}$ and $16 \mathrm{~nm}$, as confirmed by transmission electron microscopy; the VIS absorption maxima are at $545 \mathrm{~nm}, 530 \mathrm{~nm}, 525 \mathrm{~nm}$ and $519 \mathrm{~nm}$, respectively.

The gold NP coating of SU-8 surface started with a silanization step as described in section 3.3. The cured APTES-coated layer was then incubated with as prepared gold suspensions at RT for 5,20 or 60 min and finally rinsed with milliQ water. NP binding is facilitated by the electrostatic 
interaction between the negatively charged gold and the positively charged amino groups of APTES (Liu et al. 2005; Balint et al. 2009). The presence and status of the NP coating was verified by scanning electron microscopy and by VIS absorption spectroscopy.

Alternatively TPP structures (Fig. 11(d)) were coated with gold NPs using PEG-diamine linker processed as in section 3.2.2. The PEGylated surface was heated to $65^{\circ} \mathrm{C}$ for $20 \mathrm{~min}$, allowed to cool to RT, washed and dried. Prior to application the gold colloid solutions were concentrated by centrifugation by about $10 \mathrm{X}$ and dropped onto the structures covering an area of about $1 \mathrm{~cm}$ diameter. Table 3 summarizes the sample treatment and their relevant characteristics. The structures with tips (types E3 and E4 on Fig. 11(d)) were always coated with PEG-diamine linker and incubated with the $80 \mathrm{~nm}$ gold NPs for $6 \mathrm{~h}$. The flat structures (types E1, E2 and F on Fig. 11(d)) were incubated with the $80 \mathrm{~nm}$ gold for the following durations: APTES linker: $10 \mathrm{~min}, 30$ min (sample names: A10m, A30m in Table 3, respectively); PEG-diamine linker: 5 min, 10 min, $20 \mathrm{~min}, 30 \mathrm{~min}$ and $6 \mathrm{hr}$ (sample names: P5m, P10m, P20m, P30m and P6h, respectively). Additionally, one flat enhancer sample was treated with $1 \%$ acetic acid for 10 min after the PEGdiamine coating and before the $6 \mathrm{hr}$ incubation in the $80 \mathrm{~nm}$ gold NP solution (sample name P6hA).

The substrate with the NP-coated enhancers was immersed into a 1\% BSA in PBS solution and the enhancers were removed mechanically and transferred into a home-made reservoir. The bottom surface of this reservoir was formed by a fluorescent protein-coated coverslip (section 3.3). The enhancers settled onto the fluorescent layer forming the arrangement depicted on Fig. 13. The flat type E1, E2 and F structures were also coated with $41 \mathrm{~nm}$ and $16 \mathrm{~nm}$ diameter gold NPs. The surface density of the gold NPs was determined by scanning electron microscopic imaging (Hitachi S-4700). The extinction spectra of the NP-coated type F blocks were measured on a home-made microspectroscopic unit consisting of an upright microscope (Olympus BX43), a white LED light source, an $80 \mu \mathrm{m}$ diameter multimode fiber and a spectrometer (QE6500, Ocean Optics, USA).

\subsection{Characterization of SU-8 surfaces}

The relative amount of the residual epoxide was checked by measuring the IR spectra of UVpolymerized SU-8 layers and that of adjacently positioned TPP microblocks by an FTIR spectrophotometer (IFS 66, Bruker, Germany). The TPP sample was produced with the multiple polymerization of $15 \times 15 \times 6 \mu \mathrm{m}$ blocks that filled up an area of $1 \times 1 \mathrm{~mm}$ (fill factor: $\sim 85 \%$ ). 
The IR absorption spectra measured on the UV-polymerized and on the TPP samples were normalized using the treatment-unaffected $1608 \mathrm{~cm}^{-1}$ band of SU-8 (Tseng et al. 2004). The amount of residual epoxide groups was compared by the area of the band at $914 \mathrm{~cm}^{-1}$, characteristic for the epoxy ring vibration (Tseng et al. 2004).

The acid treatment of the SU-8 surfaces is the first crucial step and its various effects were evaluated. First, the presence of the surface -OH groups was confirmed on UV-polymerized SU8 surfaces (dose: $340 \mathrm{~mJ} / \mathrm{cm}^{2}$ ) with water contact angle measurements (Tao et al. 2006; Marie et al. 2006) and the hydroxyl group density was quantified with the toluidine blue method. The contact angle measurement was performed without and with acid-treatment (incubation times: 30 min and $2 \mathrm{hr}$ ), according to standard protocols (OCA20 Data Physics Instrument GmbH, Germany). For the quantification of hydroxyl groups (Tao et al. 2008) the UV-polymerized layers were incubated in the acid for $30 \mathrm{~min}, 2 \mathrm{hr}$ or $4 \mathrm{hr}$ and rinsed with milliQ water. Next, the slides were incubated with $0.1 \%$ toluidine blue in PBS (pH 7.4) for 10 min. After water rinsing the toluidine blue was desorbed from the surface by $2 \mathrm{~mL}$ of $10 \mathrm{wt}$. \% acetic acid and the VIS absorption of the collected solution was measured. The amount of toluidine blue originally bound to the SU-8 was calculated from the optical density measured at $633 \mathrm{~nm}\left(\varepsilon_{633}=4.8 \times 10^{4} \mathrm{~cm}^{-1} \mathrm{M}^{-1}\right)$ and converted to surface density of $-\mathrm{OH}$ groups assuming a 1:1 ratio between $-\mathrm{OH}$ and bound toluidine blue.

To examine the eroding effect of the acid treatment on the sub micrometer features of TPPmicrostructures a series of bars were polymerized parallel to the substrate between and outside of two supports (Fig. 16(b-e)). The bars of the very same structure were imaged before and after 30 min, $2 \mathrm{hr}$ and $4 \mathrm{hr}$ acid treatments by optical microscopy and checked for damage.

The surface morphology of untreated, acid-treated and streptavidin-coated SU-8 layers made by UV-polymerization was characterized using atomic force microscopy (AFM) (Asylum MFP-3D, Asylum Research, USA), the samples were compared through the roughness values.

The determination of protein density on the SU-8 surface was carried out in a custom built single molecule scanner based on a conventional inverted epi-fluorescence microscope (Axiovert 200 M, Zeiss, Germany) (Hesch et al. 2009; Hesse et al. 2006; Hesse et al. 2004). The DyLight650 and Alexa 568 conjugated, surface-bound streptavidin was illuminated at $647 \mathrm{~nm}$ by a $\mathrm{Kr}^{+}$laser and at $514 \mathrm{~nm}$ by an Ar+-laser respectively; the emitted fluorescence was collected onto a back- 
illuminated CCD camera (NTE/ CCD-1340/100-EMB, Roper Scientific, USA) by a 100x oil immersion objective (NA=1.45, $\alpha$ Plan-Fluar, Zeiss).

\subsection{Fluorescence enhancement and reflectivity measurements}
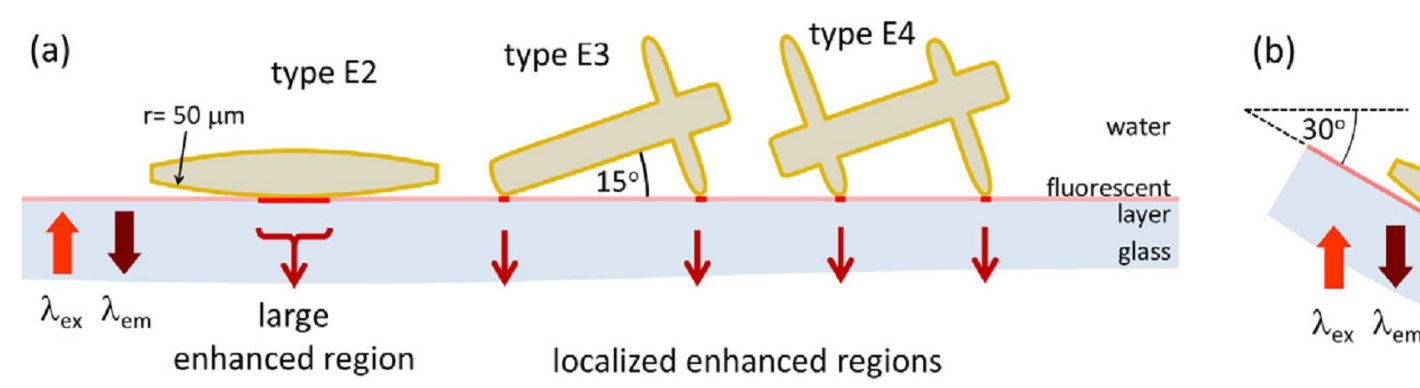

Figure 13. Arrangement of three types of enhancers over a fluorescent layer with the expected localizations of the enhancement. (a) The direction of the excitation and emission is along an axis perpendicular to the fluorescent layer. (b) Both the structures and the layer are tilted by $30^{\circ}$.

The fluorescence enhancement and reflectance measurements were carried out on an inverted laser scanning confocal microscope (LSCM, Olympus Fluoview FV 1000) with an Olympus LUMPLFL $60 \mathrm{X} \mathrm{W}$ objective, $\mathrm{NA}=0.9$ and a $10 \mathrm{x}$ objective, $\mathrm{NA}=0.4$. The schematics of the fluorescence excitation and observation and the arrangement of the gold NP-coated enhancer structures on top of the fluorescent layer are shown on Fig. 13. For DyLight 650 we used a 633 $\mathrm{nm}$ laser for excitation (power: $30 \mu \mathrm{W}$ ), observation above $645 \mathrm{~nm}$ and for Cy3 a $543 \mathrm{~nm}$ excitation laser (power: $100 \mu \mathrm{W}$ ) and observation above $555 \mathrm{~nm}$. For the quantification of the fluorescence enhancement we used data collection parameters (PMT voltage, offset and gain) where the detected signal is linear with the emitted intensity; it was verified prior to the measurements with fluorescence intensity standards. We recorded Z-stacked intensity images of the fluorescent layer with the NP-coated enhancers positioned on top of them; we always chose that slice for evaluation, where the fluorescence measured away from the enhancers was the highest. The maximum enhancement was calculated as the ratio of the intensity around the maximum intensity on the image and that of the background fluorescence. The enhancement was evaluated with the Image J software.

The reflectivity of the NP-coated structures was measured at the excitation wavelength using type F blocks immersed in water facing towards the measuring objective. The measurement was carried out on the same LSCM system, with the same data collection parameters, except the 
detected wavelength range was set to be around $633 \mathrm{~nm}$. The reflection from an Al (Aluminium) mirror $(\mathrm{R}=97 \%)$ was used as reference.

\subsection{Surface functionalization of K562 cells}

For efficient binding of cells to STA coated 3D microstructures it was required to coat them with biotin. It was done according to a supplier-provided protocol using sulfo-NHS-Biotin linker. Shortly, the cells were centrifuged and washed twice with ice cold HEPES ringer buffer ( $\mathrm{pH} 7.4)$. Then freshly prepared biotin $(1 \mathrm{mg} / \mathrm{mL}$ in HEPES) solution was added to the cells and was incubated for 20 mins on ice. Further the cells were washed with HEPES buffer to remove unbound biotin and finally the required amount of HEPES buffer containing 1\% BSA was added. Approximately $60 \mu \mathrm{L}$ from the final biotinylated cell suspension was injected into a reservoir (Secure-Seal Hybridization chamber, S24733, Thermo Fisher Scientific, USA) that has a glass cover slip bottom. Then the collected and STA functionalized polymer microstructures with $\sim 3$ $\mu \mathrm{L}$ solution were added to the cell suspension. Approximately $20 \%$ of the cells remained floating within the first 1 hour of the subsequent trapping experiments the rest bound to the bottom.

\subsection{Indirect optical trapping of cells}

The optical tweezers system was the same as in (Di Leonardo et al. 2012) . It is a holographic optical trap (HOT) system built on a Zeiss Axio Observer A1 inverted microscope with a continuous wave fiber laser $(\lambda=1070 \mathrm{~nm}, 100 \mathrm{~mW}$ at the objective, IPG-YLM-10, IPG Photonics) as a light source, an Olympus UPlanSApo water immersion objective $(60 \mathrm{X}, \mathrm{NA}=1.2)$ as a focusing element, a motorized microscope stage (Märzhäuser, Germany) for sample translation and a spatial light modulator (PLUTO NIR, Holoeye, Germany) to generate multiple traps. The optical path included a half wave plate to rotate the orientation of the plane of polarization of the beam in order to orient the trapped crosses. In order to clarify the necessity of the functionalization and to determine the binding efficiency of the functionalized TPP structures to the cells we performed several attachment tests. In these the trapped crosses or ellipsoids were trapped with steady traps and by moving the sample stage they approached the cells and were pushed against them for up to $2 \mathrm{~s}$. We did not specifically control the speed of approach, which was approximately $0.5-1 \mu \mathrm{m} / \mathrm{s}$. Then the optical trap was retracted and checked if the structure remained in the trap or remained attached to the cells. These tests were performed with and 
without cell biotinylation and with and without microstructure functionalization with STA as presented in Table 4.

A lower limit for the binding strength between the cell and the microstructure was given by performing a viscous drag experiment when a cell-attached ellipsoid was trapped and translated with a known speed (between 7 and $15 \mu \mathrm{m} / \mathrm{s}$ ) relative to the medium. Approximating the cell with a sphere, the drag force acting on it due to viscosity can be calculated by the equation (1). Provided that the couple remained in the trap during the translation, the calculated viscous force is smaller than the force that keeps the couple together. Eventually, we also used a complex OTtools with four spheroids to indirectly trap and translate cells within the sample chamber. The flat probe part of such a trapped structure was approached by a cell and attached to it. Next, the sample chamber was translated along all three directions at constant speed and the position of the cell-structure couple was evaluated by video microscopy. 


\section{Results and discussions}

\subsection{Characterization of SU-8 surface functionalization}

\subsubsection{Evaluation of the residual epoxy groups in SU-8 layers}

The relative amount of the remaining epoxide groups after SU-8 crosslinking in the UV-, and TPP-polymerized structures was compared by FTIR spectroscopy (Fig. 14). Since the functionalization is based on surface epoxy ring opening, this value resulted by the two kinds of illuminations had to be as similar as possible. The UV-polymerized layers were identically prepared except the UV light dose was varied between 170 and $16320 \mathrm{~mJ} / \mathrm{cm}^{2}$. The area of the $914 \mathrm{~cm}^{-1}$ band in the IR spectra of the polymerized SU-8 layers was compared to that of the unexposed SU-8 layer (considered as unity). The band area in the TPP spectrum was 0.446 , in the spectrum of $170 \mathrm{~mJ} / \mathrm{cm}^{2}$ dose it was 0.464 , for $510 \mathrm{~mJ} / \mathrm{cm}^{2}$ it was 0.318 and it further decreased at higher dose values. The layers exposed with $170 \mathrm{~mJ} / \mathrm{cm}^{2}$ dose, often peeled off during further treatments therefore we used $340 \mathrm{~mJ} / \mathrm{cm}^{2}$ dose to model the functionalization of TPP surfaces.

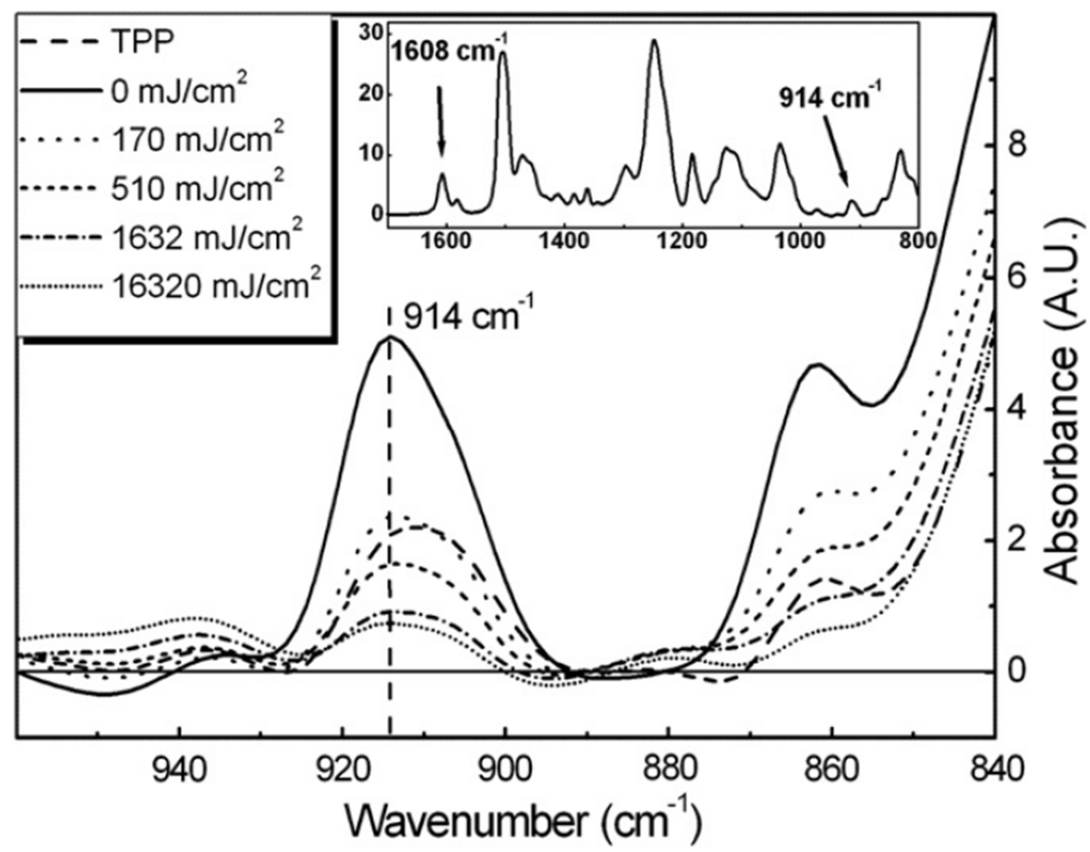

Figure 14. FTIR spectra of SU-8 layers: un-polymerized $(0 \mathrm{~mJ} / \mathrm{cm} 2)$ and polymerized by UV illumination $(170-16320 \mathrm{~mJ} / \mathrm{cm} 2)$ and two-photon absorption. The spectra are normalized with the $1608 \mathrm{~cm}-1$ band. The band at $914 \mathrm{~cm}-1$ is characteristic to the epoxide group vibration. Inset: FTIR spectrum of cured SU-8. 


\subsubsection{Effect of acid treatment}

The appearance of $-\mathrm{OH}$ groups on the SU-8 surface due to acid treatment renders it hydrophilicity as followed by contact angle measurement (Fig. 16(a)): the angle decreased from the original $82^{\circ}-42^{\circ}$ after $2 \mathrm{hr}$ of acid incubation. The surface density of hydroxyl groups, quantified by the toluidine blue method, increased almost tenfold with the incubation time (Fig. 16(a)) from $0.39 \times 10^{-9}$ to $3.89 \times 10^{-9} \mathrm{~mol} / \mathrm{cm}^{2}$ (or from $2.34 \times 10^{5}$ to $2.33 \times 10^{6} \mathrm{groups} / \mu \mathrm{m}^{2}$ ). Interestingly when tested the effect of acid incubation time on streptavidin surface density at longer incubation times smaller fluorescent intensity signal was observed, showing an eventual smaller amount of streptavidin on the surface (data not shown; the result is similar to that of (Tao et al. 2008)). In other words, larger -OH group surface density seems to decrease the eventual protein surface density.
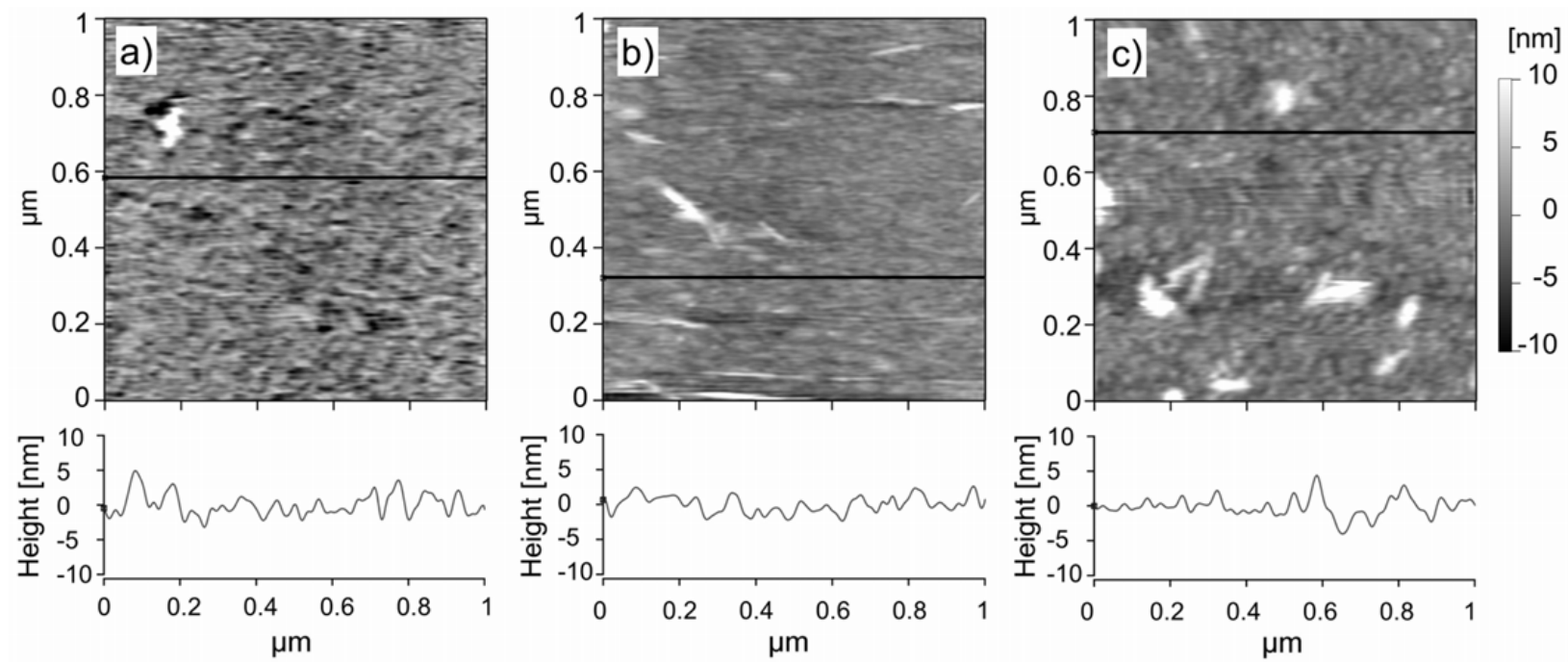

Figure 15. Surface morphology of UV-polymerized SU-8 layers determined by AFM imaging. The layers were (a) untreated, (b) incubated with acid-CAN mixture for $30 \mathrm{~min}$ and (c) incubated with acid, APTES, sulfo-NHS biotin and streptavidin.

The possible damaging effects of the acid treatment on SU-8 surface morphology and on twophoton polymerized microstructures were explored with AFM measurements on flat SU-8 surfaces (Fig. 15) and with test bars of submicrometer thickness (Fig. 16. (b-e)). The AFM measurements discovered practically unchanged surface morphology: the roughness of the untreated SU-8 is $2.6 \mathrm{~nm}$, that of the $30 \mathrm{~min}$ acid treated is $1.8 \mathrm{~nm}$ and that of the final, streptavidin treated surface is $2.5 \mathrm{~nm}$. It means that further protein or gold NP incubation steps will find the same smooth SU-8 surface after acid treatment as it was before. As illustrated by the 
representative image of TPP test bars on Fig. 16(c) the 30 min acid treatment causes no damage on these fragile sub-micrometer features. After $2 \mathrm{hr}$ treatment, however, the free hanging parts of the bars suffer disfiguration: bend or disappear completely (encircled region Fig. 16(d-e)). The 4 $\mathrm{hr}$ incubation completely removed the TPP structures from the supporting glass surface. This finding together with the fact that prolonged acid treatment decreases the final protein density justified that 30 min acid treatment was sufficiently long; therefore it was used in the subsequent functionalization experiments.
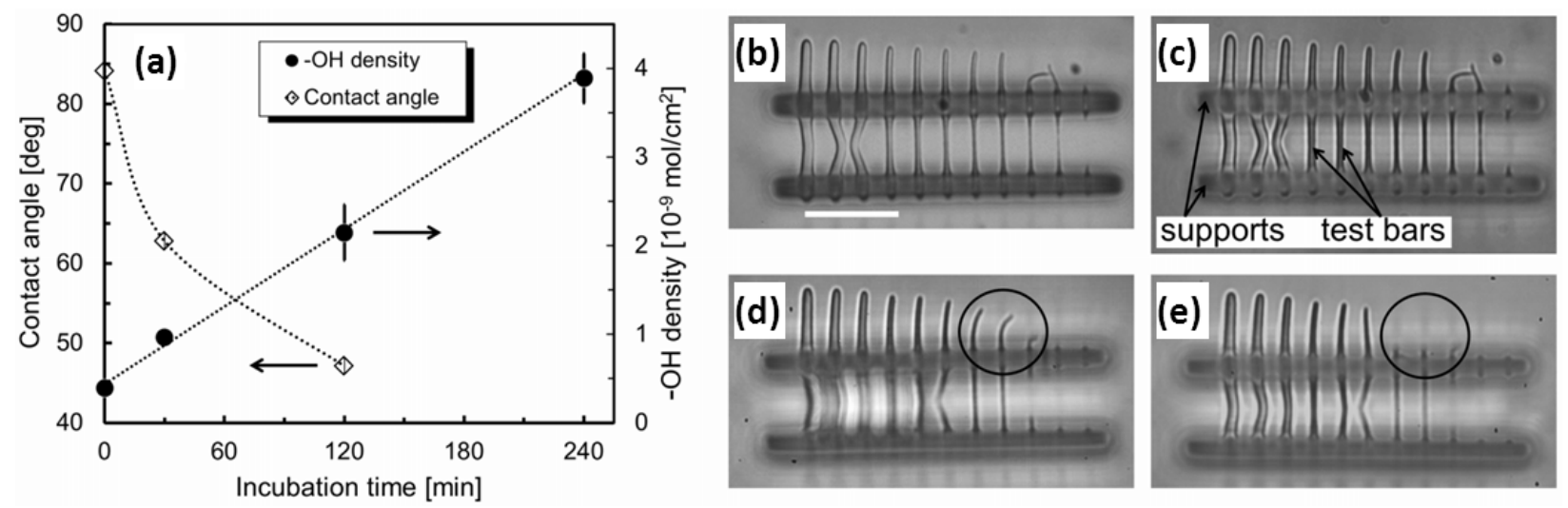

Figure 16. Effects of the acid treatment on UV- and two-photon polymerized SU-8 structures. (a)

Surface - $\mathrm{OH}$ density and contact angle change with the acid incubation time measured on UVpolymerized layers. Lines are used to guide the eye. Optical microscopic images of a TPP test structure (b) before and (c) after 30 min acid treatment. Another TPP test structure (d) before and (e) after $2 \mathrm{hr}$ acid treatment. Note the missing lines after $2 \mathrm{hr}$ acid treatment (encircled). Scale bar: 10 $\mu \mathrm{m}$.

\subsubsection{Quantification of protein density on $\mathrm{SU}-8$ surface}

\subsubsection{Streptavidin coating with silanization}

The graph on Fig. 17(a) shows the streptavidin surface density measured on UV-polymerized layers after each functionalization step and that on TPP structures (in the form of $15 \times 15 \times 6 \mu \mathrm{m}$ blocks) after all the treatments. The comparison of the treatment methods (Table 1) illustrates that Mthd 4 yields the highest protein density: about $4544 \pm 520 \mathrm{STA} / \mu \mathrm{m}^{2}, 1-2.5$ orders of magnitude higher than the other methods. This relation shows the importance of carrying out all the steps for an effective protein coating. The biotinylated surface without APTES coating (Mthd 3) gives 15x smaller surface density $\left(294 \pm 32 \mathrm{STA} / \mu^{2} \mathrm{~m}^{2}\right.$; here sulfo-NHS-biotin probably binds unspecifically to the surface, to which STA can subsequently bind. It is also noteworthy that the 
bare SU-8 (no treatment at all, Mthd 0) can bind 7.6 and 3.3 times more streptavidin molecules than acid only (Mthd 1) or acid plus APTES (Mthd 2) treated samples. This phenomenon, similar to that reported by Blagoi et al.(Blagoi et al. 2008), can be explained by the reduction of the number of available epoxide groups. It is known that primary amines, for instance those on the surface of proteins, can react with epoxide groups (Marie et al. 2006). The opening of the epoxide groups during acid treatment reduces the number of available reactants: less protein finds epoxide to bind to. The TPP structures, when receiving all the treatments (Mthd 4) show the highest streptavidin surface density $\left(8156 \pm 1496 \mathrm{STA} / \mu \mathrm{m}^{2}\right)$. The small difference, between the protein surface densities on UV- and TPP surfaces justifies our choice of the model system for the TPP microstructures.

It is evident that in the case of TPP structures also Mthd 4 provides the highest protein density on the surface. The treatment was successfully applied on micrometer-sized spirals with line thickness of submicrometer scale (Fig. 17(c)). The background signal, i.e. the fluorescent protein bound to the supporting glass, for Mthd 0, Mthd 3 and Mthd 4 is practically the same as the signal on SU-8 (Fig. 17(a)), although it means a weak selectivity between SU-8 and glass, it does not affect the method's applicability on the optical trapping microtools as these are made to be removed from the substrate and transferred to a microfluidic environment for further use. Such fluorescent streptavidin coated OT-tools are shown on Fig. 17(b) after being removed from its original substrate and transferred to a microfluidic chamber.

From the obtained protein surface density the arrangement of the streptavidin molecules can be assessed considering each streptavidin molecule as a $5 \pm 1 \mathrm{~nm}$ diameter sphere (Cooper et al. 1994). The assumption of a tightly packed monolayer of streptavidin on a smooth surface results in about $23000 \pm 9000 \mathrm{STA} / \mu \mathrm{m}^{2}$ density. This means an about $20 \%$ surface coverage for the UV polymerized layer and 35\% for TPP structures. From the above data it seems that the OT-tools can be easily equipped with a layer of streptavidin that enables their use in optical tweezersactuated experiments where biotin-streptavidin interaction is to be utilized. 
(a)

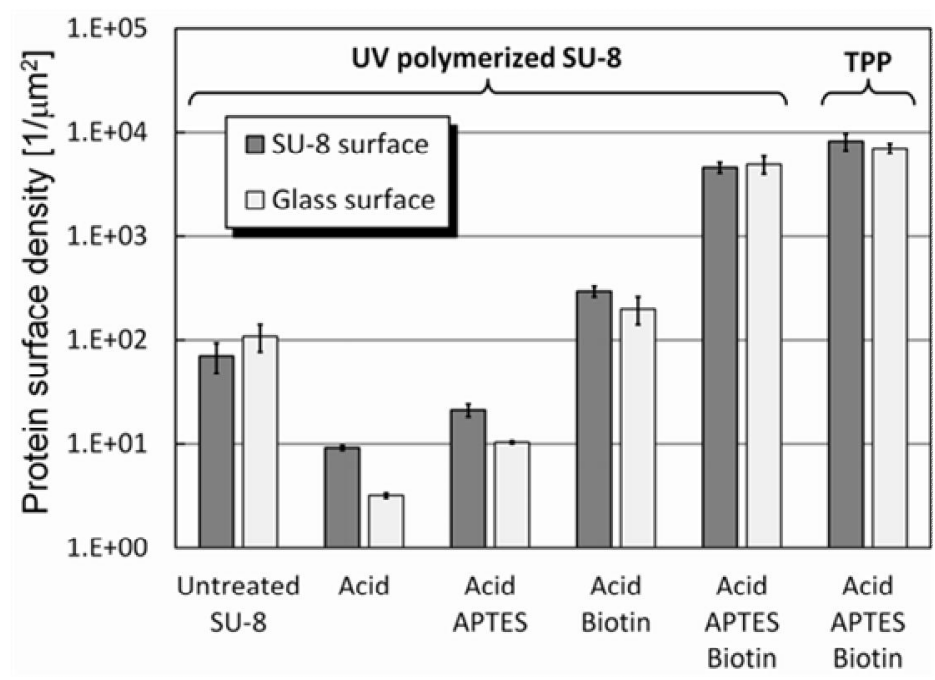

(b)

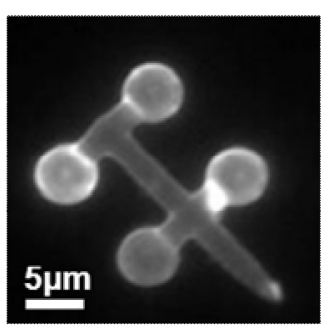

(c)

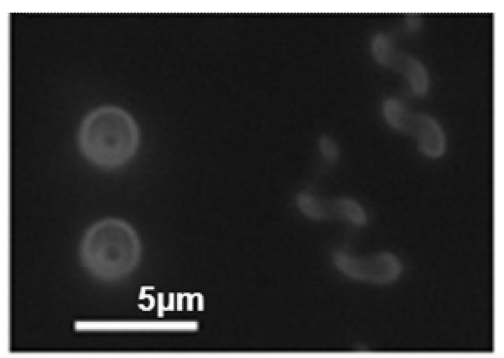

Figure 17. (a) Streptavidin surface densities measured on differently treated, UV- and two-photon polymerized SU-8 surfaces and on their glass substrates. Fluorescence microscopy images of streptavidin-coated two-photon polymerized (b) OT-tools and (c) spirals, standing and lying on the substrate glass.

\subsubsection{Streptavidin and antibody coating with PEGylation}

Though the results of this section are not yet published, I believe that they fit with the line of thesis, hence they are included here.

The comparison of the treatment methods (Table 2, Fig. 18(a)) reveals that considering the biotinassisted specific binding of streptavidin Mthd 4 yields the highest protein density: it is about 1.6 $\mathrm{x} 10^{5} \mathrm{STA} / \mu \mathrm{m}^{2}$. This is orders of magnitude higher than that of the other biotin-based methods and about 20 times higher than that of the glutaraldehyde-based ones. Also, the SU-8 binds considerably more protein than the supporting glass, dissimilarly to the silane-based method. The large difference in density and the occasional inhomogeneously coated surfaces for the other methods again underlines the necessity of carrying out all the functionalization steps. In case of the biotin linker, there was no significant difference between the UV-and the TPP polymerized SU-8 surface, while for glutaraldehyde linker, the TPP structures were covered with about 5 times more streptavidin. The high streptavidin density for biotin linker shows the superiority of specific biding on to SU-8 (Mthd 4) over unspecific binding (Mthd 5).

In case of the unspecific glutaraldehyde based functionalization, Alexa 568 conjugated IgG antibody was also used besides streptavidin (Table 2). It is evident from Fig. 18(b) that the 
treatment involving all the steps (Mthd 7) gives more than 20 times higher surface protein density than the untreated SU-8 itself (Mthd 6) regardless the type of protein.
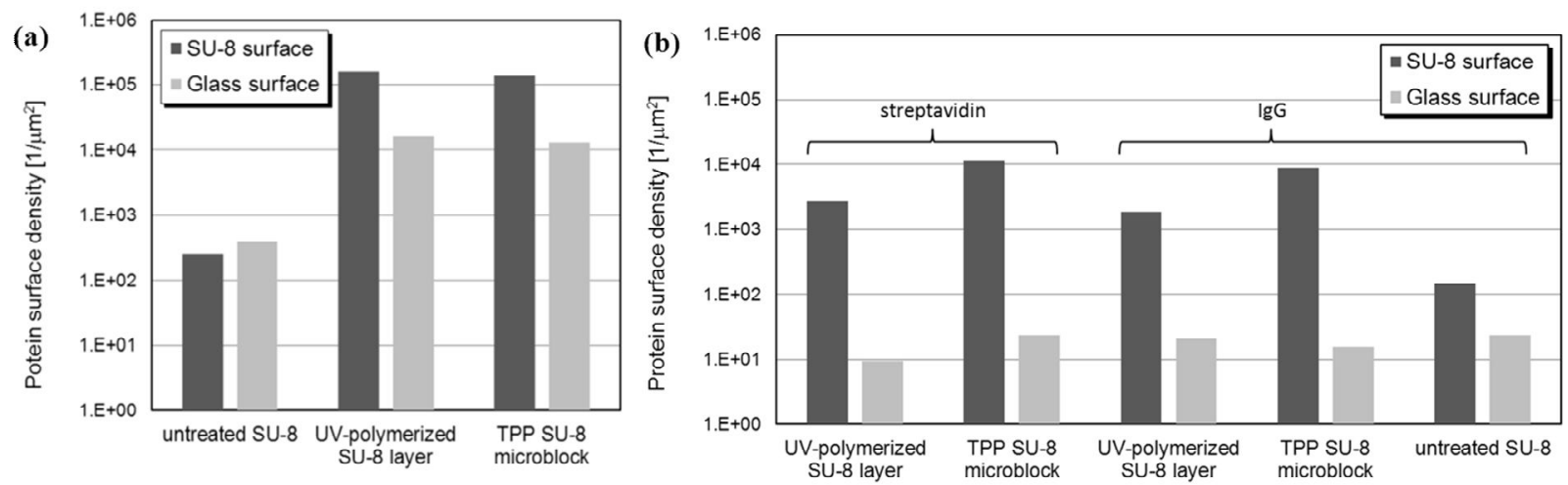

Figure 18. Streptavidin and antibody surface densities measured on differently treated, UV- and two-photon polymerized SU-8 surfaces using the PEGylation method together with (a) specific biotin linker (b) unspecific glutaraldehyde linker.

\subsubsection{Characterization of gold NPs on SU-8 layers and microstructures}

Gold particles of $24 \mathrm{~nm}$ average diameter were immobilized on SU-8 surfaces as described in section 3.4. The SEM images shown on Fig. 19(a-c) reveal the necessity of the APTES treatment for successful gold NP binding. Without it only 4 NPs on average bind onto $1 \mu \mathrm{m}^{2}$ area. With APTES treatment $5 \mathrm{~min}$ of incubation in the NP suspension resulted in $76 \pm 17 \mathrm{NP} / \mu \mathrm{m}^{2}$ density with even distribution, reaching $446 \pm 25 \mathrm{NP} / \mu \mathrm{m}^{2}$ with $60 \mathrm{~min}$ incubation (an about $20 \%$ surface coverage). The surfaces always bear a monolayer of the NPs and even at the highest packing density there is no apparent NP clustering. The VIS absorption spectra of dry, NP-coated SU-8 layer (Fig. 19(d)) has a peak at $530 \mathrm{~nm}$ and lacks any further band above $600 \mathrm{~nm}$, also indicating the absence of aggregate (Chen et al. 2011). The amplitudes of the peaks at $530 \mathrm{~nm}$ for the two incubation times further reflect the difference in surface NP density. For optical tweezers application it is crucial to have low NP density on the polymerized SU-8 microstructures to reduce scattering that hinders trapping. However, for applications such as surface enhanced Raman spectroscopy a densely packed NP layer is better. We found that controlling the incubation time the optimum NP density can be set on the TPP microstructures. Gold coating with APTES works also well on TPP structures with sub-micrometer features. Fig. 19(e) shows the tip ( $\sim 400 \mathrm{~nm}$ thickness, $\sim 150 \mathrm{~nm}$ radius of curvature) region of a NP-coated OT microtool. The even distribution of NPs with a density around $150 \mathrm{NP} / \mu \mathrm{m}^{2}(\sim 8 \%$ surface coverage $)$ is 
evident. This value is expected to be suitable for optically trapping a few $\mu \mathrm{m}$ diameter SU-8 sphere (Liu et al. 2005).
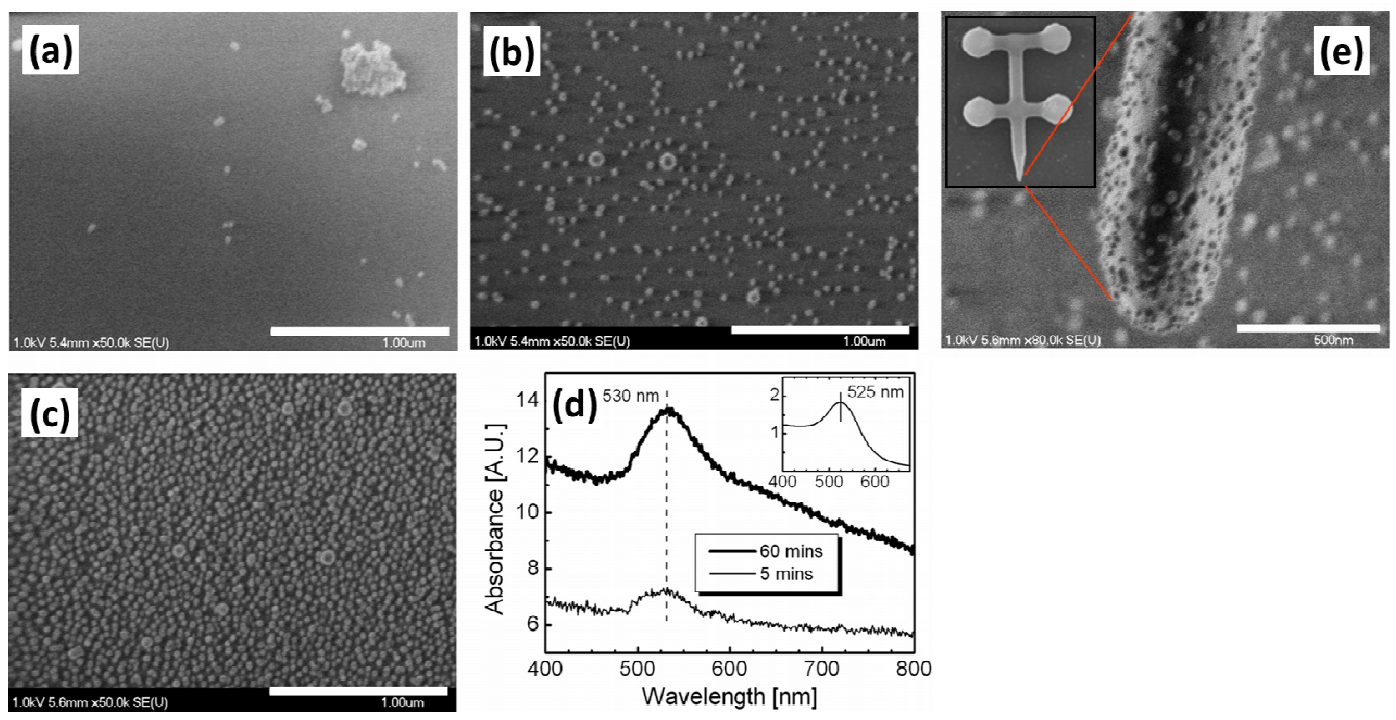

Figure 19. (a-c) Electron microscopic images of $24 \mathrm{~nm}$ gold nanoparticles (NPs) bound to flat UVpolymerized SU-8 surfaces and (d) visible absorption spectra of such layers. All the surfaces were treated with acid and (b and c) were further treated with APTES (90 min) prior to gold NP incubation. Gold incubation times were: (a, c) $60 \mathrm{~min}$ and (b) $5 \mathrm{~min}$. Scale bars: $1 \mu \mathrm{m}$. The NP incubation times for the spectra were $5 \mathrm{~min}$ (thin line) and $60 \mathrm{~min}$ (thick line). Inset: absorption spectrum of gold NP solution containing $24 \mathrm{~nm}$ diameter particles

In order to achieve higher NP surface densities, PEG-diamine linker was used. Variously coated TPP microstructures of type F were used to measure the VIS extinction spectra, the NP surface densities and reflectivity. The measured extinction spectra of the $80 \mathrm{~nm}$ diameter NP layers show a dominant band around $550 \mathrm{~nm}$, the plasmonic frequency of the gold NPs and a broader band above $650 \mathrm{~nm}$. The NP density increased from about $47 \mathrm{NP} / \mu \mathrm{m}^{2}$ (sample A10m) to about 120 $\mathrm{NP} / \mu^{2}$ (sample P6hA). This increase changes the relative amplitude of the two bands (a shoulder above $650 \mathrm{~nm}$ on Fig. 20(a) and the integrated area of the extinction spectra between $450 \mathrm{~nm}$ and $850 \mathrm{~nm}$ (an almost linear increase $\left(\mathrm{R}^{2}=0.88\right)$ on Fig. 20(d). The former change is attributed to the interaction of the dipoles of closely-spaced gold NPs (Kevin L. P et al. 1993; Balint et al. 2009). Fig. 20(b) and (c) shows very similar NP densities over different parts of a type E4 enhancer, indicating that the efficiency of the coating is independent of the surface morphology. The tip area is also coated with high density and the resulted envelope of the (Fig. $20(c))$ tip has a radius of curvature of about $350 \mathrm{~nm}$. 

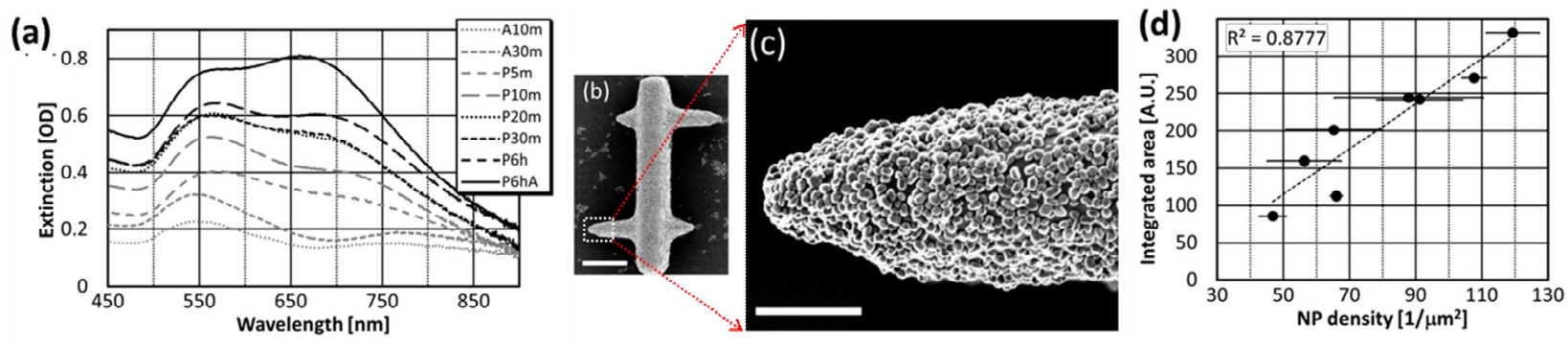

Figure 20. Characterization of $80 \mathrm{~nm}$ gold NP-coated TPP structures. (a) VIS extinction spectra of gold NP layers on type F microblocks prepared with various incubation times and linker molecules (for sample names see Table 2 ). (b) SEM images of gold NP-coated microstructure with tips (type E4). (c) Enlarged, NP-covered tip of the same structure. (d) The integrated area of the extinction spectra between $450 \mathrm{~nm}$ and $850 \mathrm{~nm}$. Scale bars: $5 \mu \mathrm{m}$ (b) and $1 \mu \mathrm{m}$ (c).

We performed reflectivity measurements on the type F microstructures (Fig. 21(a,b) that have only one surface coated with gold. 4 selected samples were used for the measurement: two APTES-linked (A10m and A30m) and two PEG-diamine linked (P5m and P6h) NP layers; the reference was an $\mathrm{Al}$ mirror $(\mathrm{R}=97 \%)$. The highest measured reflectance was $26.7 \%$ (sample $\mathrm{P} 6 \mathrm{~h}$ ) and the smallest was $6.6 \%$ (sample A10m) (Fig. 21(c)). The reflectivity measured on tilted type F structures (tilt angle $15^{\circ}$ ) was reduced to $\sim 16 \%$ of the non-tilted case (Fig. 21 (b)).
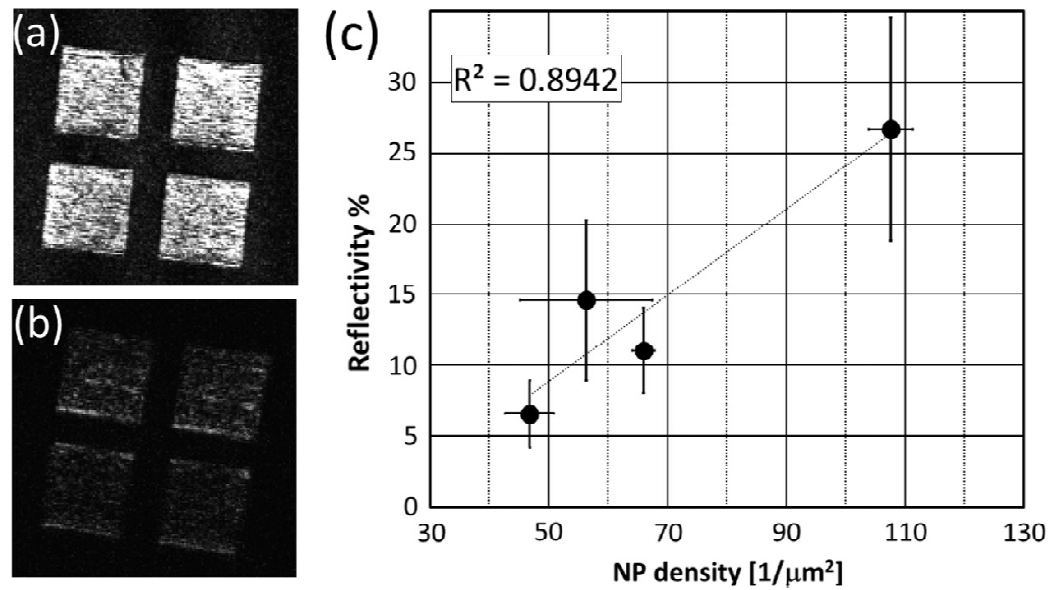

Figure 21. Reflectivity of $80 \mathrm{~nm}$ gold NP layers measured on type F microblocks. LSCM image of the four microblock with the normal of their surface parallel (a) and at $15^{\circ}$ (b) to the optical axis. (c) Correlation of the reflectivity of such coated microblocks with the NP density measured on their surfaces. 


\subsection{Application of functionalized TPP structures}

\subsubsection{Fluorescence enhancement}

We wanted to answer four specific questions regarding the fluorescence enhancement achieved by gold NP-coated TPP enhancers. One: what is the degree of localization of the enhancement that can be achieved by these structures? Two: whether the different NP densities would result in different enhancement factors? Three: can we observe enhancement in that more general case when the plane of the fluorescent layer is not perpendicular to the direction of the optical axis? Four: how different is the enhancement effect at different excitation wavelengths?

\begin{tabular}{|c|c|c|c|c|c|c|c|c|c|c|c|c|}
\hline Sample name & $\begin{array}{l}\text { Tipped } \\
\text { enhancer }\end{array}$ & A10m & A30m & P5m & P10m & P20m & P30m & P6h & P6hA & $\begin{array}{l}16 \mathrm{~nm} \\
\text { gold NP }\end{array}$ & $\begin{array}{l}41 \mathrm{~nm} \\
\text { gold NP }\end{array}$ & Cy3 \\
\hline Linker molecule & PEG-dia. & APTES & APTES & PEG-dia. & PEG-dia. & PEG-dia. & PEG-dia. & PEG-dia. & PEG-dia. & PEG-dia. & PEG-dia. & PEG-dia. \\
\hline NP diameter [nm] & 80 & 80 & 80 & 80 & 80 & 80 & 80 & 80 & 80 & 16 & 41 & 80 \\
\hline NP incubation time & $6 \mathrm{~h}$ & $10 \mathrm{~m}$ & $30 \mathrm{~m}$ & $5 \mathrm{~m}$ & $10 \mathrm{~m}$ & $20 \mathrm{~m}$ & $30 \mathrm{~m}$ & $6 \mathrm{~h}$ & $6 \mathrm{~h}$ & $6 \mathrm{~h}$ & $6 \mathrm{~h}$ & $6 \mathrm{~h}$ \\
\hline NP density (NP/ $\left./ \mu \mathrm{m}^{2}\right)$ & & $46.8 \pm 4$ & $66 \pm 1.9$ & $56.3 \pm 1$ & $65.2 \pm 14$ & $91 \pm 13$ & $87.9 \pm 22$ & $107.6 \pm 4$ & $119 \pm 8$ & & & \\
\hline $\begin{array}{l}\text { Integrated spectrum } \\
450-850 \mathrm{~nm} \text { (A.U.) }\end{array}$ & & 85.3 & 112.4 & 159.3 & 201.5 & 241.7 & 244.4 & 270.6 & 331 & & & \\
\hline $\begin{array}{l}\text { Spectral amplitude } \\
\text { at } 633 \mathrm{~nm}(\mathrm{OD})\end{array}$ & & 0.16 & 0.21 & 0.36 & 0.44 & 0.55 & 0.55 & 0.6 & 0.79 & 0.66 & 0.83 & \\
\hline Reflectance (\%) & & $6.6 \pm 2.3$ & $11 \pm 3$ & $14.6 \pm 6$ & & & & $26.7 \pm 8$ & & & & \\
\hline Applied fluorophore & $\begin{array}{l}\text { DyLight } \\
650\end{array}$ & $\begin{array}{l}\text { DyLight } \\
650\end{array}$ & $\begin{array}{l}\text { DyLight } \\
650\end{array}$ & $\begin{array}{l}\text { DyLight } \\
650\end{array}$ & $\begin{array}{l}\text { DyLight } \\
650\end{array}$ & $\begin{array}{l}\text { DyLight } \\
650\end{array}$ & $\begin{array}{l}\text { DyLight } \\
650\end{array}$ & $\begin{array}{l}\text { DyLight } \\
650\end{array}$ & $\begin{array}{l}\text { DyLight } \\
650\end{array}$ & $\begin{array}{l}\text { DyLight } \\
650\end{array}$ & $\begin{array}{l}\text { DyLight } \\
650\end{array}$ & Су3 \\
\hline $\begin{array}{l}\text { Additional } \\
\text { treatment }\end{array}$ & & & & & & & & & $\begin{array}{l}\text { Acetic } \\
\text { acid }\end{array}$ & $\begin{array}{l}\text { Acetic } \\
\text { acid }\end{array}$ & $\begin{array}{l}\text { Acetic } \\
\text { acid }\end{array}$ & \\
\hline $\begin{array}{l}\text { Fluorescence } \\
\text { enhancement factor }\end{array}$ & $\begin{array}{l}3.16 \\
\pm 0.46\end{array}$ & $\begin{array}{l}2.3 \\
\pm 0.21\end{array}$ & $\begin{array}{l}2.47 \\
\pm 0.22\end{array}$ & $\begin{array}{l}4.27 \\
\pm 0.51\end{array}$ & $\begin{array}{l}4.67 \\
\pm 0.37\end{array}$ & $\begin{array}{l}4.8 \\
\pm 0.50\end{array}$ & $\begin{array}{l}4.8 \\
\pm 0.54\end{array}$ & $\begin{array}{l}4.98 \\
\pm 0.38\end{array}$ & $\begin{array}{l}5.06 \\
\pm 0.52\end{array}$ & \begin{tabular}{|l|}
3.23 \\
\pm 0.38
\end{tabular} & \begin{tabular}{|l}
4 \\
\pm 0.46
\end{tabular} & $\begin{array}{l}3.1 \\
\pm 0.3\end{array}$ \\
\hline
\end{tabular}

Table 3: Summary of the properties of and results obtained with the prepared gold NP-coated TPP samples.

\subsubsection{Localized fluorescence enhancement}

Fig. 22(a) shows localized fluorescence enhancement achieved by a type E4 gold NP-coated microstructure over a layer of DyLight 650-conjugated strepatavidin observed with a confocal microscope. The enhancement is clearly localized only to the regions where the tips of the microstructure are in contact with the fluorescent surface. The enhancement factor, quantified by LSCM was between 2.5 and 4 with the average of $3.16 \pm 0.46$ and was restricted to an area less than $1 \mu \mathrm{m}^{2}$ (Fig. 22(b) and (d)). The slightly elongated intensity distribution is due to the elongated shape of the tip end that inherits the shape of the polymerizing voxel. Since the fluorescence signal of the fluorophore layer itself regarded homogeneous, we conclude that the 
localized fluorescence enhancement originates from the NP-fluorophore interaction and not from local concentration inhomogeneity of the fluorophore.
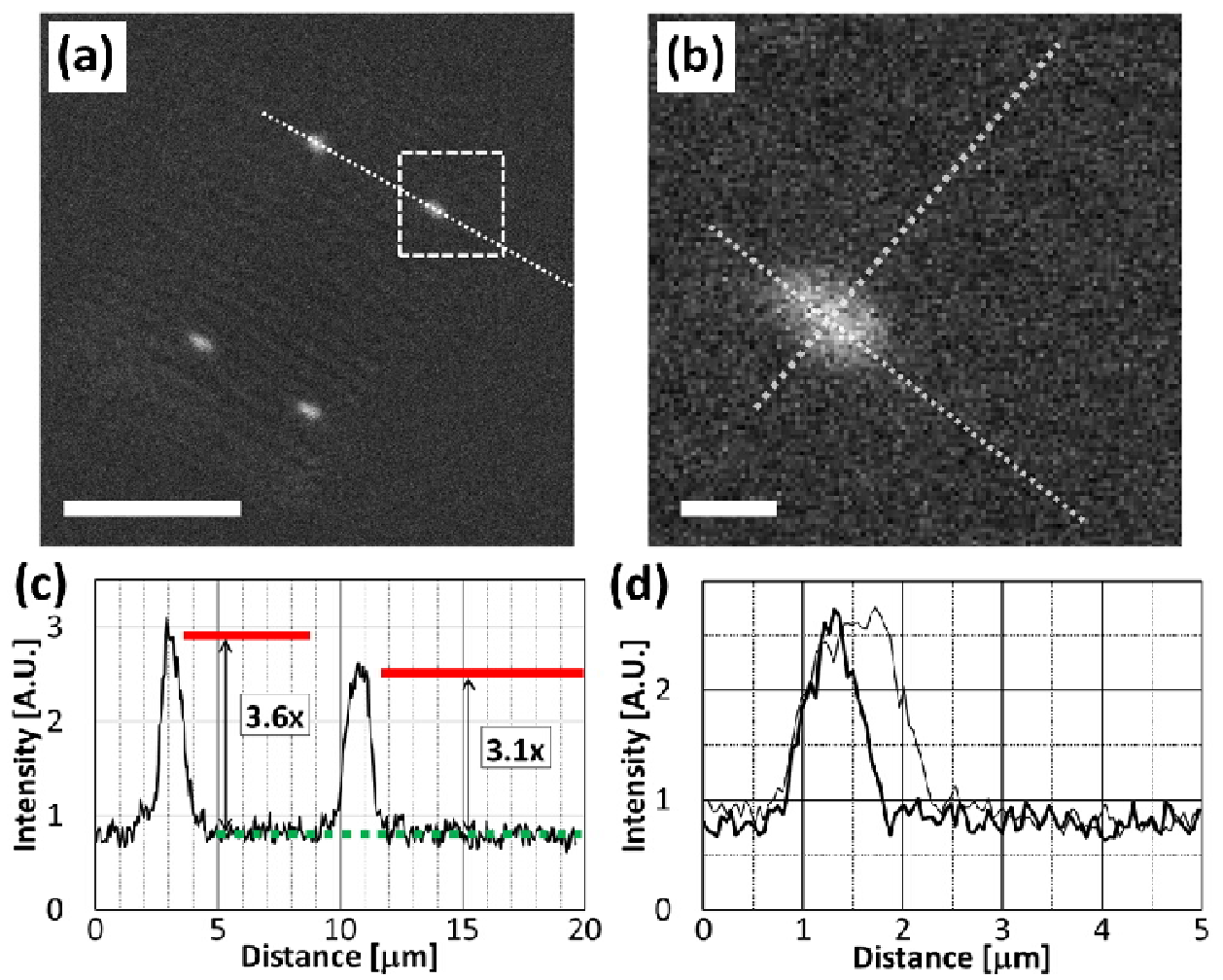

Figure 22. Localized fluorescence enhancement with tipped microstructures, coated with $80 \mathrm{~nm}$ gold NPs. Laser scanning confocal fluorescence microscopic images ((a) and (b)) of the enhanced regions of a DyLight 650 fluorophore layer. (b) Close-up of the highlighted tip on (a). (c) Intensity trace along the dashed line on (a). (d) Intensity traces along the lines on (b). Scale bars: $10 \mu \mathrm{m}$ (a) and 1 $\mu \mathrm{m}(\mathrm{b})$.

\subsubsection{Large-area fluorescence enhancement}

Fluorescence enhancement over larger area might be of interest in some applications so we also investigated this scenario. Characteristic confocal and bright-field microscopy images of three round-sided enhancer structures are shown in Fig. 23(a) and (d). Fig. 23(b) shows fluorescence enhancement patterns achieved by these enhancers at high NP density (sample P6hA). Fig. 23(e) and (f) shows quantitatively the fluorescence intensity change along the marked lines achieved by the highest (sample P6hA) and the lowest (sample A10m) NP density enhancers. The enhancement factor showed also extremals for these samples: for the highest NP-density it was $5.05 \pm 0.58$ and for lowest it was $2.3 \pm 0.2$. Regarding the whole range of NP densities, the enhancement factor depends on the extinction of the NP layer at the excitation wavelength, 633 
$\mathrm{nm}$, in a saturating manner as shown in Fig. 23(c). The dependence of the fluorescent intensity on the lateral position for each structure is related to the varying distance between the fluorescent layer and the structure's coated surface; its origin is discussed later. Type E1 and E2 non-coated microstructures were used as controls; the enhancement factor was lower than 1.3 and the periodic intensity lines were also typical.
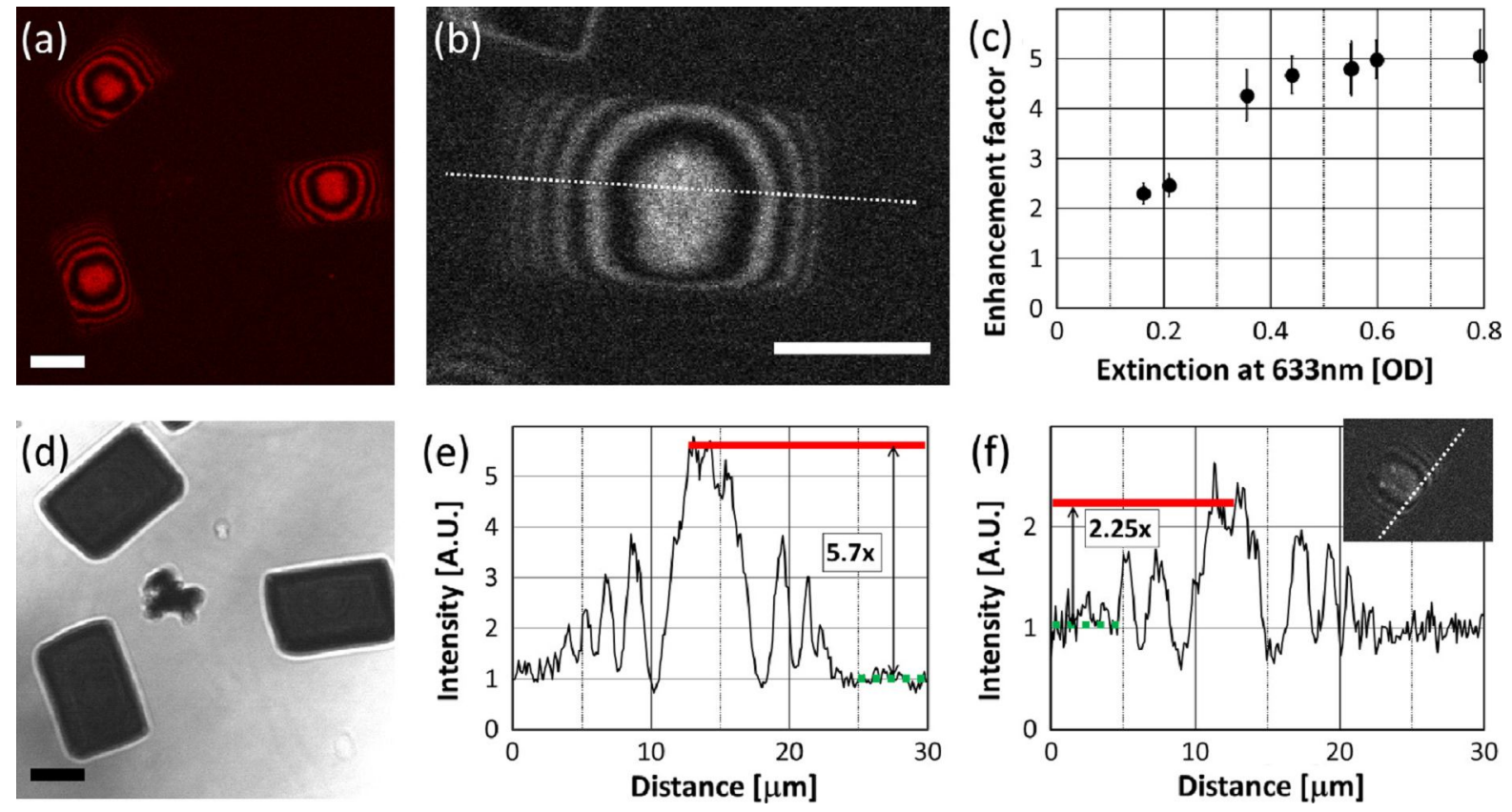

Figure 23. The effect of NP surface density on the degree of fluorescence enhancement. LSCM images of a DyLight 650 fluorophore layer with round-sided enhancers, sample P6h ((a) and (b)). (d) Bright-field microscopy image of the same structures as on (a). (c) Dependence of the enhancement factor on the amplitude of the extinction spectrum at $633 \mathrm{~nm}$. (e) Intensity trace along the dashed line on (b). (f) Intensity trace along the line on the inserted image measured with a round-sided enhancer, sample A10m. Scale bars: $10 \mu \mathrm{m}$.

We further studied the fluorescent enhancement in two kinds of tilted arrangement with high gold NP density samples (P6h) over DyLight 650 fluorophore (Fig. 24). First, we measured enhancement with type E3structures, where the NP-coated surfaces are tilted by $15^{\circ}$ in respect to the fluorophore layer, which is itself perpendicular the observation. The most important feature here is that each structure displays a series of intense fluorescent lines with an average distance of maxima of $990 \pm 67 \mathrm{~nm}$ (Fig. 23(e)). In the second scenario the fluorescent surface and the enhancer surface were in contact and parallel, but both were tilted relative to the observation by 
$30^{\circ}$ (Fig. 24(a, d)). A representative slice from the recorded Z-stack series of the LSCM data is seen in Fig. 24(a) showing an enhancement between 3 and 4 (Fig. 24(d)).

The fluorescence enhancement was also demonstrated with smaller diameter gold NPs. We coated both type E1 and E2 flat enhancer structures with $16 \mathrm{~nm}$ and $41 \mathrm{~nm}$ diameter NPs, using the procedure resulting in samples P6hA. The amplitude of the extinction spectra of the smaller NPs (Fig. 24(c)) is of comparable amplitude to that of the $80 \mathrm{~nm}$ ones, but the weight of the plasmonic absorption band around $550 \mathrm{~nm}$ seems to decrease with NP diameter. The enhanced

fluorescence of smaller sized NP coatings shows the same distance-dependent periodic intensity pattern (Fig. 24(f)) as seen for the $80 \mathrm{~nm}$ NPs. The measured enhancement factors are $3.23 \pm 0.38$ for the $16 \mathrm{~nm}$ and $4 \pm 0.46$ for the $41 \mathrm{~nm}$ NP layer. In comparison, the enhancement qualitatively obeys the same characteristics for all three NP sizes but the enhancement factor values are the highest for the $80 \mathrm{~nm}$ NPs. We further determined the enhancement for Cy3 fluorophore, the absorbance of which overlaps with the gold NP plasmonic absorption band ( $\sim 550 \mathrm{~nm})$. For these measurements the excitation wavelength was at $543 \mathrm{~nm}$ and we used high NP density (P6h). The achieved 3.1 \pm 0.27 enhancement factor was about 1.6 times smaller than observed in the red region; the structured fluorescence observation was also present.

\subsubsection{On the origin of the fluorescence enhancement}

As it was mentioned earlier the fluorescence enhancement occurred in a spatially structured manner. For type E2 (round-sided) structures the maximum observed intensity was underneath the center and the second maximum was at around $5 \mu \mathrm{m}$ from it laterally. The NP layer to fluorophore distance at this point is about $250 \mathrm{~nm}$ and the enhancement is between 3 or 4 (Fig. 23(e)). For the tilted structures, the periodic lines of enhanced intensity are present for fluorophore-NP layer distance of several micrometers (Fig. 24(e)). In addition, in the darker regions between the enhanced ones the fluorescence is often weaker than the background. In other words, those parts of the enhancers also increase fluorescence where the NP layer to fluorophore layer distance is larger than what is required for plasmonic enhancement $(<50 \mathrm{~nm})$. Therefore we assume that the origin of the enhancement is primarily the formation of standing waves similar to examples reported earlier (Kiessling et al. 2003). Standing waves are formed by the interference of the incoming excitation beam and the excitation beam reflected by the lower gold NP layer (Fig. 24(b)). Enhancement is observed when the interference maximum (antinode) is on the fluorescent layer, while at the interference minima (node) the fluorescence intensity 
decreases even below the background level. This phenomenon is exploited in Fluorescence Interference Contrast microscopy (FLIC). Our reflecting surface-fluorophore-illumination arrangement (see Fig. 13(a)) is very similar to that of FLIC.

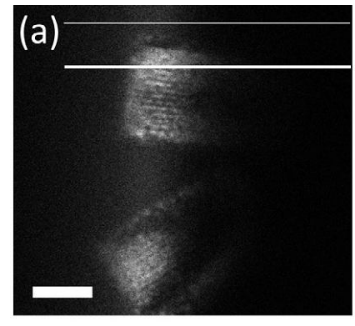

(d)

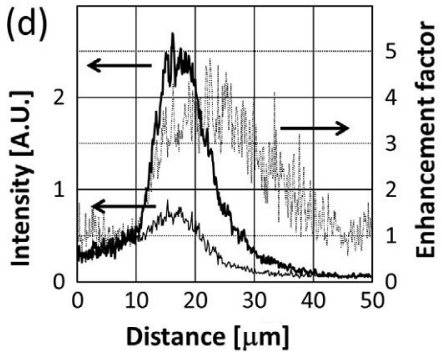

(b)

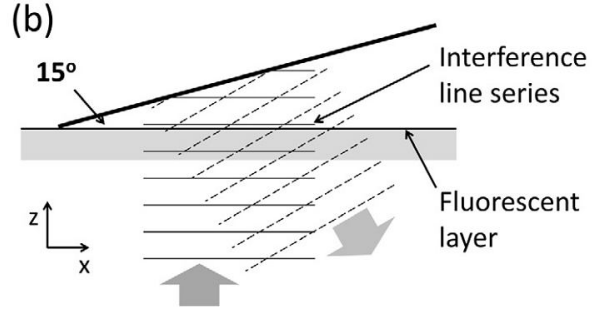

(e)

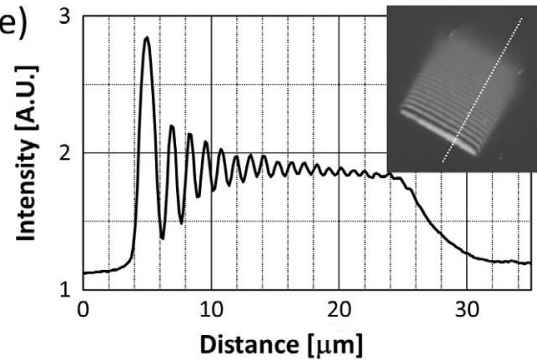

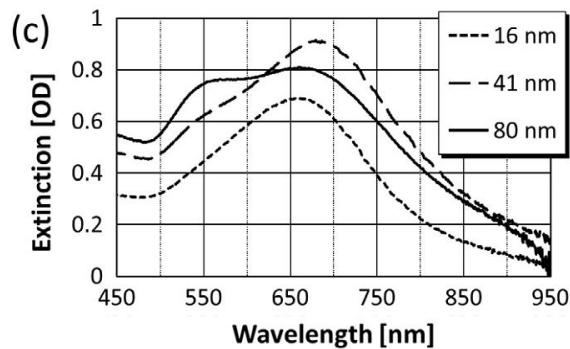

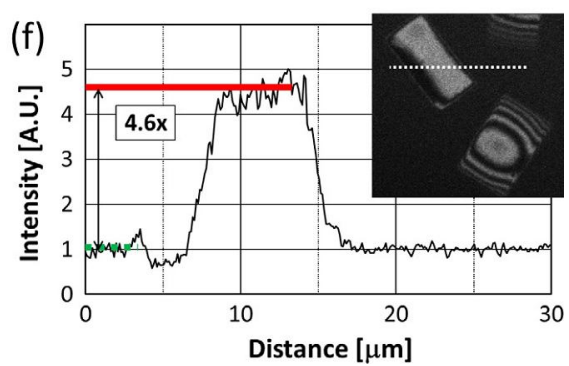

Figure 24. Fluorescence enhancement by tilted enhancers and by small diameter NP layers. (a) Laser scanning fluorescence microscopic images with NP-coated enhancers over a DyLight 650 fluorophore layer that was tilted by $30^{\circ}$ (sample P6h, types E1 and E2). (b) Illustration of the formation of standing waves with $15^{\circ}$ tilted reflective enhancer structure over fluorescent layer. (c) VIS extinction spectra of gold NP layers with NPs of three different diameters on type F structures (sample P6hA). (d) Intensity traces along the marked lines on (a). Thick line corresponds to the thick line on (a), thin line corresponds to the thin line on (a) and the dotted line is the enhancement profile.

(e) Intensity trace along the dashed line on the inserted image showing enhanced fluorescence measured with a type E3 enhancer. (f) Intensity trace along the dashed line on the inserted image showing enhanced fluorescence measured with a type E1 and E2 enhancers coated with $41 \mathrm{~nm}$ diameter gold NPs.

The observed fluorescence enhancement due to standing wave formation is explained as follows. Assuming plane waves for the excitation and $0^{0}$ incidence angle for the reflection, the intensity increase in the interference maxima compared to the incoming beam is $(1+\sqrt{R})^{2}$ times where $R$ is reflectivity. In our arrangement standing waves are generated by the reflection from the lower surface of the enhancers. We measured reflectivity values on type F structures at $633 \mathrm{~nm}$ to be between $6.6 \%$ and $26.7 \%$ and assumed that the same values are valid for the type E1 and E2 enhancers. This result in 1.58-2.3 times higher intensities in the interference maxima than that of 
the incoming wave. This excitation light intensity increase alone cannot explain the florescence enhancement factor of 2.3-5. It is plausible to assume that the emitted light is also reflected by the enhancers. We propose that the emitted light that propagates towards the observer and the one that is reflected by the enhancer to the same direction can also undergo interference. Similarly to our findings, the reflection of both the excitation and emitted light is considered as the source of enhancement as in (Le Moal et al. 2007). Le Moal and co-workers found a periodic enhancement pattern, similar to ours, with characteristic distances dictated by the excitation and emission wavelength. We have to note that interference of the emitted light of broad emission spectrum can only take place close to the reflective surface due to the short coherence length of the light (expected within $4 \mu \mathrm{m}$ ). Therefore, from those parts of the enhancers that are closer to the fluorescent layer than $\sim 1 \mu \mathrm{m}$, emitted light interference is expected to be efficient. In summary we suggest that the intensity increase due to the interference of the excitation as well as the emitted light is responsible for the observed fluorescence enhancement. Plotting the enhancement factor as the function of the square of the intensity increase resulted by and calculated from the measured reflectivity, a linear trend with a slope close to unity is obtained (Fig. 25). This supports that the reflection of the excitation and emitted light both contributes to the enhancement.

The presence of standing waves can also be concluded from the fluorescence data measured on the enhancers with a reflective surface tilted by $15^{\circ}$ (type E3 on Fig. 13(a)). Due to reflection from a tilted plane surface, (Fig. 24(b) and (e)), an interference line series of equal distance is expected and observed. Taking into account the $\sim 15^{\circ}$ tilt, the propagation direction of the two interfering beams makes $150^{\circ}$, therefore the interference line distance $\Delta \mathrm{x}$ along the fluorescent layer can be calculated:

$$
\Delta x=\frac{\lambda}{n \cdot \sin (\Theta)}
$$

where $\lambda$ is the wavelength, $n$ is the refractive index of the medium and $\Theta$ is the angle of the relative propagation directions. The periodicity $\Delta \mathrm{x}$ of the lines on these images was measured to be $990 \pm 67 \mathrm{~nm}$. From this, the wavelength of the beams that produced the line series was calculated to be $658.5 \pm 44.7 \mathrm{~nm}$, which is almost exactly halfway between the excitation wavelength and the emission peak of the fluorophore. This agreement further supports the assumption of the standing waves. 


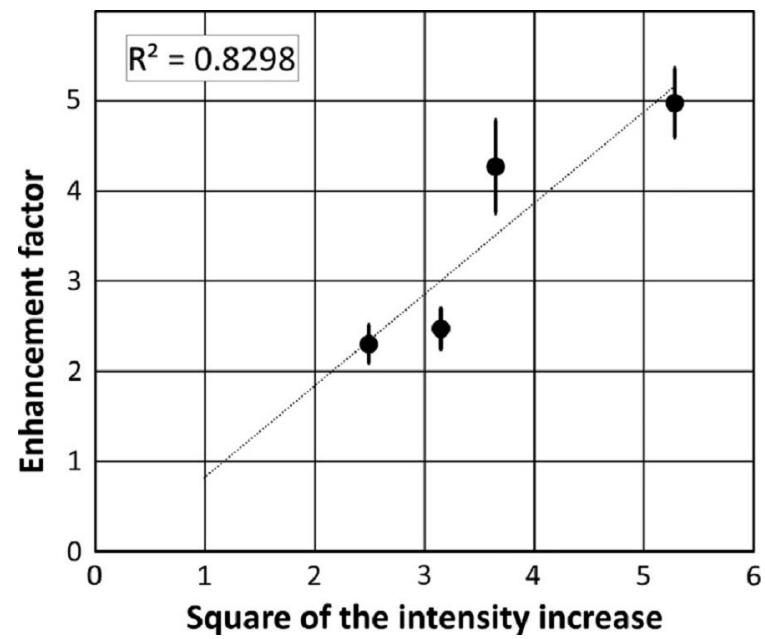

Figure 25. Correlation of the enhancement factor measured on four samples of different gold NP densities with the square of the intensity increase in the interference maxima. The intensity increase was calculated from the measured reflectivity of the corresponding four samples..

Finally we cannot rule out that plasmonic enhancement also plays a role in the enhancement at parts of the enhancers where the NPs are sufficiently close $(10-30 \mathrm{~nm})$ to the fluorophore layer. This phenomenon is probably located to small clusters of NPs which are somewhat elevated from the surrounding monolayer of NPs. These clusters are located to areas smaller than the optical resolution of our LSCM system; therefore this effect cannot be conclusively detected.

The observed enhancement factors fall in the range of 2-6 which is slightly smaller than that of plasmonic enhancement (Stranik et al. 2005; Anger et al. 2006), but is in the range of the reflective type of enhancement (Kim et al. 2006; Choumane et al. 2005; Le Moal et al. 2007). This factor could be improved along the following two separate paths. The mechanism is governed by the reflectance of the metal layer, which could be improved either by increasing the NP density or coating the enhancers by a continuous thin metal layer (Le Moal et al. 2007). To improve the plasmonic enhancement one could consider (i) preparing nonspherical nanoparticles (nanorods, nanostars (Mohamed et al. 2000; Kumar et al. 2008), (ii) increasing the surface density of the NPs to reduce the interparticle distance(Zin et al. 2009) or (iii) using nanopatterned metal arrangement on the surface of the enhancers (Pompa et al. 2006). 


\subsubsection{Indirect cell manipulation}

\subsubsection{Binding efficiency of cells to TPP structures}

For efficient indirect trapping of live cells with TPP structures it is crucial to achieve strong linkage in a reproducible manner that also forms fast between the two components. In our yet unpublished experiments we performed attachment tests on the cells with structures coated with streptavidin using PEG-diamine and biotin linker. The primary structures we used were crosses (we also used ellipsoids) to illustrate that cells can be manipulated with structures being more complex than a microbead and can display contact areas of different size. The target K562 cells were selected randomly, being either freely floating or substrate-attached. Fig. 26(a-f) shows a series of snapshots of the approach, attachment and translation of a cell with a cross structure. We regarded the attachment successful if the trap could not remove the structure from the cell surface any more. For substrate-attached cells it meant that the trap lost the structure and for freely floating cells the couple was dragged along when the channel was translated (with approx. 10 $\mu \mathrm{m} / \mathrm{s}$ speed).

The success rate for the various functionalization combinations can be seen on Table 4 . When the streptavidin-coated structures touched the biotinylated cells, the success rate was $88.4 \%$ for crosses and was $97.5 \%$ for ellipsoids; when the cells were not biotinylated, this number fell to $30 \%$, showing some intrinsic binding ability of the streptavidin to the cell membrane. When the TPP structures were not coated with the protein, the success rate was always below $10 \%$.

\begin{tabular}{lllll} 
& \multicolumn{2}{l}{ Biotinylated cells } & \multicolumn{2}{l}{ Non-biotinylated cells } \\
\hline Streptavidin-coated crosses & $88.4 \%$ & $(\mathrm{~N}=43)(97.5 \%$ for ellipsoids $)$ & $30 \%$ & $(\mathrm{~N}=20)$ \\
\hline Non-coated crosses & $7.5 \%$ & $(\mathrm{~N}=53)$ & $2.5 \%$ & $(\mathrm{~N}=40)$
\end{tabular}

Table 4: Binding efficiency of TPP structures to cells with various functionalization combinations.

\subsubsection{Indirect trapping of cells}

The ellipsoids or crosses could not be removed from substrate-attached cells anymore, because the strength of the binding between streptavidin and biotin is higher than the maximum optical force our instrument can exert on them; the rupture force is more than $100 \mathrm{pN}$ for a single biotinstreptavidin linkage (Wong et al. 1999). Drag-force experiments were performed with indirectlytrapped cells translated in the medium at known speeds. Using drag speeds of $10 \pm 5 \mu \mathrm{m} / \mathrm{s}$ with cells of $15 \pm 5 \mu \mathrm{m}$ diameter, drag forces between 1.2 and $2.4 \mathrm{pN}$ were calculated. We did not 
observe trapped structure-cell detachment during the drag experiments. The restoring force acting on the ellipsoids during the drag was independently determined. From the measured trap stiffness (between 13 and $28 \mathrm{pN} / \mu \mathrm{m}$ ) and ellipsoid displacements (Fig. 26(g) displacement histogram along $\mathrm{X}$ axis) restoring force up to $7 \mathrm{pN}$ was obtained; it is slightly higher than calculated with the drag force method. The possible reason is that the cells are not elevated from the substrate surface, which imposes an extra friction force on them during drag.
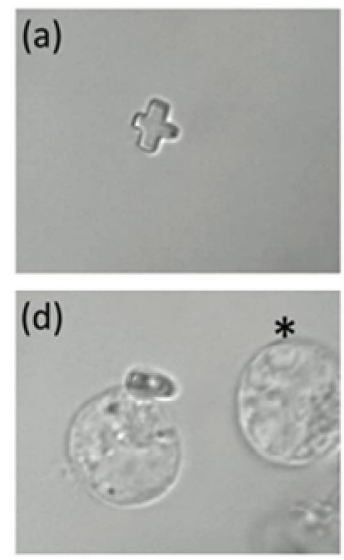
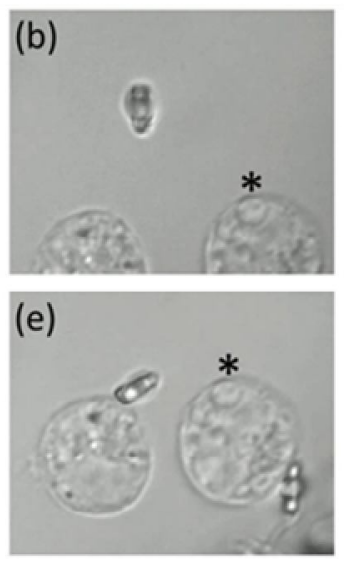
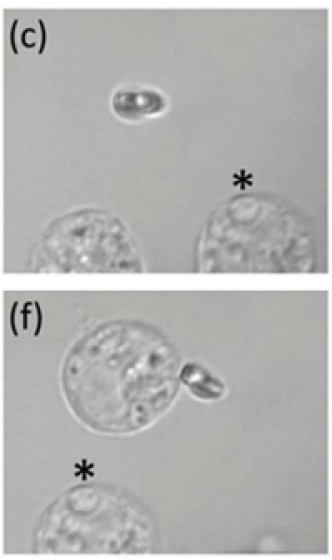

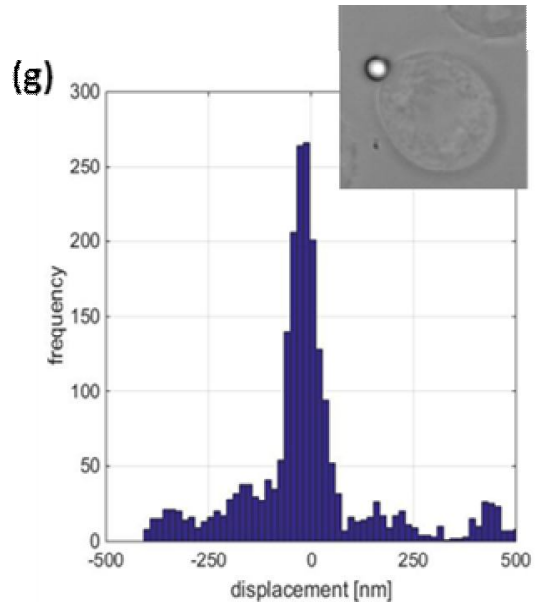

Figure 26. Attachment and optical manipulation of a live K562 cell with a two-photon polymerized, streptavidin-coated cross. (a) The cross before being trapped. (b) The trapped cross. (c) The trapped cross rotated with the trap by $90^{\circ}$. (d) The trapped cross is pushed against the cell. (e) The cell being dragged around the reference cell (*). (f) The cell passed the reference cell. (g) Histogram showing the displacement of an optically trapped ellipsoid attached to a cell from its equilibrium position during the drag of the couple

To further demonstrate the applicability of two-photon polymerized structures for indirect cell trapping and manipulation we constructed a complex structure to be used in a multitrap system. This structure consists of a handle and a probe part and can be trapped with a set of four static optical traps. The main advantages of using such a complex structure for manipulation are the increased distance of the cell and the very intense trapping beam and the possibility of 6 degrees of freedom manipulation. According to our knowledge this is the first demonstration of a twophoton polymerized, optically trapped, complex (non-spherical) structure chemically modified in a specific way to manipulate a living cell. Fig. 27 shows snapshots of the translation of such a structure with a cell attached to it perpendicular to their long axis (scan speed approx. $14 \mu \mathrm{m} / \mathrm{s}$ ) 
highlighting the effect of the viscous drag. During the scan a tilt was observed and measured to be 4.8 degrees.
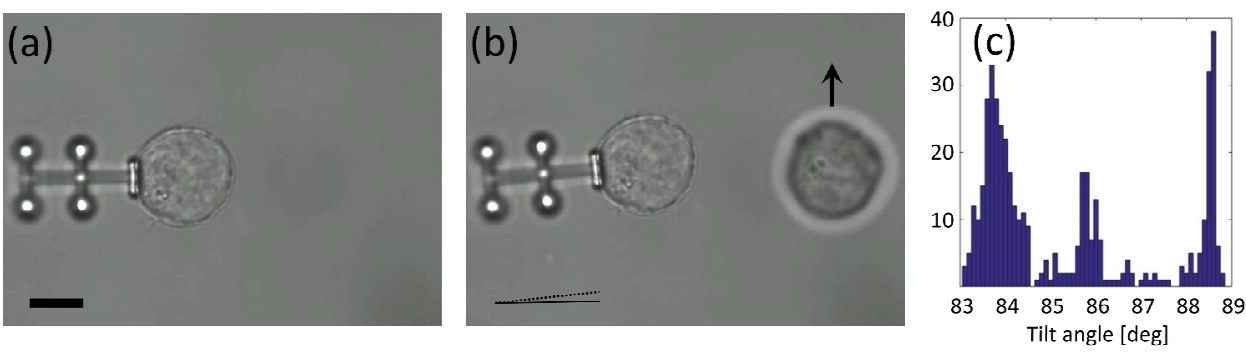

Figure 27. Indirect optical manipulation of a live K562 cell with a complex two-photon polymerized, streptavidin-coated manipulator. (a) The cell is attached to the structure in a motionless environment. (b) The sample chamber is translated along the direction of the arrow that inflicts a small tilt of the couple. (c) Histogram of the tilt angles relative to the drag direction (steady-state angle: $88.5^{0}$ ). Scale bar: $10 \mu \mathrm{m}$. 


\section{Summary}

This study presents insights into different surface functionalization methods of SU-8 photoresist based 3D microstructures and their possible biological applications.

First, a functionalization with the protein streptavidin and gold NPs is shown that is based on CAN-catalyzed acid and subsequent aminosilane treatments. The straightforward method can be safely applied on any arbitrarily shaped microstructure because the careful choice of the duration of the acid incubation preserves their integrity including sub-micrometer sized free-standing features and surface morphology. The protein surface density results show that a protein layer with about $20-35 \%$ surface coverage is formed on the surface of the TPP structures, rendering them applicable in optical tweezers experiments where biotin-streptavidin interaction is to be utilized. Evenly distributed gold NPs with controlled density were formed on the structural elements of the microstructures by adjusting a simple parameter, the incubation time. The NP density is a key condition in the further applications such as optical trapping and surface enhanced Raman enhancement. The potential of TPP to make 3D sub-micrometer structure together with the presented functionalization process can hold a great promise and can serve as a platform towards biological systems and application.

Secondly it was successfully demonstrated that TPP can indeed be effectively used to make novel microcarriers with shape variability for metal NP layers to be used for fluorescence enhancement. It was shown that such NP-coated TPP microstructures can efficiently enhance fluorescence detection. Fluorescence enhancement was localized to less than $1 \mu \mathrm{m}^{2}$ area with a factor of more than 3. The microstructures could also enhance fluorescence even by a factor of 6 when larger areas were considered. The enhancement factor was found higher when NPs were applied at higher surface density. It was also dependent on the excitation wavelength of the fluorophore and is not limited to surfaces perpendicular to the optical axis of the excitation/detection. It is believed that the origin of the enhancement is mainly the formation of standing waves due to the reflection of the excitation field as well as the emitted light by the NP layer; plasmonic effect in the close contact areas may also play a role in the enhancement. The application of polymer microstructures as enhancers offers the possibility to combine fluorescence enhancement with optical tweezers in the future.

Lastly indirect optical manipulation of live cells was presented. In order to enable the manipulation with motions more complex than what can be achieved by a microbead, special- 
shaped intermediate objects are needed. Furthermore, the structures are needed to be functionalized efficiently to realize quick and stable cell-microstrcture binding. We showed that using a PEG-diamine based coating method the protein layer on the 3D microtools has ten times higher surface density than that achieved with the APTES-based method. It provides enough protein for a quick microstructure-cell attachment. Binding efficiency of about $90 \%$ was achieved with such-coated microstructures and a basic but successful indirect manipulation of live cells with optically trapped, protein-functionalized microtools was demonstrated. 


\section{References}

Amato L, Gu Y, Bellini N, Eaton S, Cerullo G and Osellame R (2012). Integrated threedimensional filter separates nanoscale from microscale elements in a microfluidic chip. Lab on a Chip 12: 1135-1142.

Amiot C L, Xu S, Liang S, Pan L and Zhao J X (2008). Near-infrared fluorescent materials for sensing of biological targets. Sensors 8: 3082-3105.

Anger P, Bharadwaj P and Novotny L (2006). Enhancement and quenching of single-molecule fluorescence. Physical Review Letters 96: 113002-113006.

Anker J N, Hall W P, Lyandres O, Shah N C, Zhao J and Van Duyne R P (2008). Biosensing with plasmonic nanosensors. Nature Materials 7: 442-453.

Ashkin A (1970). Acceleration and trapping of particles by radiation pressure. Physical Review Letters 24: 156-159.

Balint S, Kreuzer M P, Rao S, Badenes G A, Miskovsky P and Petrov D ( 2009). Simple route for preparing optically trappable probes for surface enhanced raman scattering. Journal of Physical Chemistry C 113: 17724-17729.

Balslev S, Mironov A, Nilsson D and Kristensen A (2006). Micro-fabricated single mode polymer dye laser. Optics Express 14: 2170-2177.

Bianchi S and Di Leonardo R (2010). Real-time optical micro-manipulation using optimized holograms generated on the GPU. Computer Physics Communications 181: 1444-1448.

Blagoi G, Keller S, Johansson A, Boisen A and Dufva M (2008). Functionalization of SU-8 photoresist surfaces with IgG proteins. Applied Surface Science 255: 2896-2902.

Bustillo J M, Howe R T and Muller R S (1998). Surface micromachining for microelectromechanical systems. Proceedings of the IEEE 86: 1552-1574. 
Campbell S (2001). The Science and Engineering of Microelectronic Fabrication. New York, Oxford Univ. Press, .

Chen J Y and Chen Y C (2011). A label-free sensing method for phosphopeptides using twolayer gold nanoparticle-based localized surface plasma resonance spectroscopy. Analytical and Bioanalytical Chemistry 399: 1173-1180.

Chen Y S, Tal A and Kuebler S M (2007). Route to Three-dimensional metallized microstructures using cross-linkable epoxide SU-8. Chemistry of Materials 19: 38583860 .

Choumane H, Ha N, Nelep C, Chardon A, Reymond G O, Goutel C, Cerovic G, Vallet F, Weisbuch C and Benisty H (2005). Double interference fluorescence enhancement from reflective slides: Application to bicolor microarrays. Applied Physics Letters 87: 031102.

Cooper J M, Shen J, Young F M, Connolly P, Barker J R and Moores G (1994). The imaging of streptavidin and avidin using scanning tunnelling microscopy. Journal of Materials Science: Materials in Electronics 5: 106-110.

Crivello J V (1999). The discovery and development of onium salt cationic photoinitiators. Journal of Polymer Science A 37: 4241-4254.

Dai W and Wang W (2008). Fabrication of comb-drive micro-actuators based on UV lithography of SU-8 and electroless plating technique. Microsystem Technologies 14: 1745-1750.

Delamarche E, Donzel C, Kamounah F S, Wolf H, Geissler M, Stutz R, Schmidt-Winkel P, Michel B, Mathieu H J and Schaumburg K (2003). Microcontact printing using poly(dimethylsiloxane) stamps hydrophilized using poly(ethylene oxide)-silanes. Langmuir 19: 8749-8758.

Deubel M, Wegener M, Linden S, Freymann G and John S (2006). 3D-2D-3D photonic crystal heterostructures fabricated by direct laser writing. Optics Letters 3: 805-807. 
Di Leonardo R, Buzas A, Kelemen L, Vizsnyiczai G, Oroszi L and Ormos P (2012). Hydrodynamic synchronization of light driven microrotors. Physical Review Letters 109: $034104-034108$.

Drexhage K H (1970). Influence of a dielectric interface on fluorescence decay time. J. lournal of Luminescence 1-2: 693-701.

Dufresne E R and Grier D G (1998). Optical tweezer arrays and optical substrates created with diffractive optical elements. Review of Scientific Instruments 69: 1974-1977.

Dufresne E R, Spalding G C, Dearing M T, Sheets S A and G G D (2001). Computer-generated holographic optical tweezer arrays. Review of Scientific Instruments 72: 1810-1816.

Durr N J, Larson T, Smith D K, Korgel B A, Sokolov K and Yakar A B (2007). Two-photon luminescence imaging of cancer cells using molecularly targeted gold nanorods. Nano Letters 7: 941-945.

El-Ali J, Perch-Nielsen I R, Poulsen C R, Bang D D, Telleman P and Wolf A (2004). Simulation and experimental validation of a SU-8 based PCR thermocycler chip with integrated heaters and temperature sensor. Sensors and Actuators A 110: 3-10.

El-Sayed I H, Huang X H and A E-S M (2005). Surface plasmon resonance scattering and absorption of anti-EGFR antibody conjugated gold nanoparticles in cancer diagnostics: applications in oral cancer. Nano Letters 5: 829-834.

Fischer J and Wegener M (2013). Three-dimensional optical laser lithography beyond the diffraction limit. Laser \& Photonics Reviews 7: 22-44.

Formanek F, Takeyasu N, Tanaka T, Chiyoda K, Ishikawa A and Kawata S (2006). Threedimensional fabrication of metallic nanostructures over large areas by two-photon polymerization. Optics Express 14: 800-809.

Forth S, Sheinin M, Inman J and M W (2013). Torque measurement at the single molecule level. Annual Review of Biophysics 42: 583-604. 
Franziska K, Thomas S, Joachim F, Zhongxiang J, Clemens M F, Georg v F, Martin W and Martin B (2010). Elastic fully three-dimensional microstructure scaffolds for cell force measurements. Advanced Materials 22: 868-871.

Frimat J-P, Becker M, Chiang Y, Janasek D, Hengstler J G, Franzke J and West J (2011). A microfluidic array with cellular valving for single cell co-culture. Lab on a Chip 11: 231237.

Galajda P and Ormos P (2002). Rotation of microscopic propellers in laser tweezers. Journal of Optics B-Quantum and Semiclassical Optics 4: S78-S81.

Gartia M R, Hsiao A, Sivaguru M, Chen Y and Liu G L (2011). Enhanced 3D fluorescence live cell imaging on nanoplasmonic substrate. Nanotechnology 22: 365203-365214.

Geddes C D (2013). Metal-enhanced fluorescence. Physical Chemistry Chemical Physics 15: 19537.

Gelorme J D, Cox R J and Gutierrez S A R (1989). Photoresist composition and printed circuit boards and packages made there with. U. S. Patent.

George O (1981). Principles of polymerization. New York Wiley Interscience.

Gittard S, Nguyen A, Obata K, Koroleva A, Narayan R and Chichkov B (2011). Fabrication of microscale medical devices by two-photon polymerization with multiple foci via a spatial light modulato. Biomedical Optics Express 2: 3167-3178.

Gittard S and R N (2010). Laser direct writing of micro and nano-scale medical devices. Expert Review of Medical Devices 7: 343-356.

Goldys E (2009). Fluorescence Applications in Biotechnology and Life Sciences,. Hoboken, Wiley-Blackwell.

Haes A J and Van Duyne R P (2002). A nanoscale optical biosensor: sensitivity and selectivity of an approach based on the localized surface plasmon resonance spectroscopy of triangular silver nanoparticles. Journal of the American Chemical Society 124: 10596-10604. 
Hesch C, Hesse J, Jacak J and Schütz G J (2009). Two-stage focus hold system for rapid ultrasensitive readout of large area biochips. Journal of Microscopy 234: 251-254.

Hesse J, Jacak J, Kasper M, Regl G, Eichberger T, Winklmayr M, Aberger F, Sonnleitner M, Schlapak R, Howorka S, Muresan L, Frischauf A M and Schütz G J (2006). RNA expression profiling at the single molecule level. Genome Research 16: 1041-1045.

Hesse J, Sonnleitner M, Freudenthaler G, Jacak J, Höglinger O, Schindler H and Schütz G (2004). Single-molecule reader for high-throughput bioanalysis. Analytical Chemistry 76: 5960-5964.

Hilario J and Kowalczykowski S C (2010). Visualizing protein-DNA interactions at the singlemolecule level. Current Opinion in Chemical Biology 14: 15-22.

Hong G, Tabakman S M, Welsher K, Chen Z, Robinson J T, Wang H, Zhang B and Dai H (2011). Near-infrared-fluorescence-enhanced molecular imaging of live cells on gold substrates. Angewandte Chemie International Edition 50: 4644-4648.

Houseman B T, Huh J H, Kron S J and Mrksich M (2002). Peptide chips for the quantitative evaluation of protein kinase activity. Nature Biotechnology 20: 270-274.

Iranpoor N and Salehi P (1995). Ceric ammonium nitrate: a mild and efficient reagent for conversion of epoxides to b-nitrato alcohols. Tetrahedron 51: 909-912.

Jaeger C (2002). Introduction to Microelectronic Fabrication. New Jersey Prentice Hall.

Jenness N, Wulff K, Johannes M, Padgett M, Cole D and Clark R (2008). Three-dimensional parallel holographic micropatterning using a spatial lightmodulator. Optics Express 16: 15942-15948.

Kato J, Takeyasu N, Adachi Y, Sun H-B and Kawata S (2005). Multiple-spot parallel processing for laser micronanofabrication. Applied Physics Letters 86: 044102.

Kelemen L, Valkai S and Ormos P (2006). Integrated optical motor. Applied Optics 45: 27772780 . 
Kelemen L, Valkai S and Ormos P (2007). Parallel photopolymerisation with complex light patterns generated by diffractive optical elements. Optics Express 16: 14488-14497.

Keller S (2008). Fabrication of an autonomous surface stress sensor with the polymer SU-8. PhD-Thesis Technical University of Denmark.

Kevin L. P and W G M (1993). Adsorption of proteins onto surfaces containing end-attached oligo( ethylene oxide): A model system using self-assembled monolayers. Journal of the American Chemical Society 115: 10714-10721.

Khandurina J and Guttman A (2002). Bioanalysis in microfluidic devices. Journal of Chromatography A 943: 159-183.

Kiessling V and Tamm L K (2003). Measuring distances in supported bilayers by fluorescence Interference-contrast microscopy: Polymer supports and SNARE proteins. Biophysical Journal 84: 408-418.

Kim J Y and Osterloh F (2006). Planar gold nanoparticle clusters as microscale mirrors. Journal of the American Chemical Society 128: 3868-3869.

Kovacs G T A, Maluf N I and Petersen K E (1998). Bulk micromachining of silicon. Proceedings of IEEE 86: 1536-1551.

Kumar P S, Pastoriza-Santos I, Rodriguez-Gonzalez B, De Abajo F J G and Liz-Marzan L M (2008). High-yield synthesis and optical response of gold nanostars. Nanotechnology 19: 015606 .

LaBianca, Nancy C, Gelorme and Jeffrey D (1995). High-aspect-ratio resist for thick-film applications. Proceedings of the SPIE 2438: 846-852.

Lakowicz J R (2001). Radiative decay engineering: biophysical and biomedical applications. Analytical Biochemistry 298: 1-24. 
Le Moal E, Fort E, Le've^que-Fort S, Cordelie` P, Fontaine-Aupart M-P and Ricolleau C (2007). Enhanced fluorescence cell Imaging with metal-coated slides. Biophysical Journal 92: $2150-2161$.

Liesener J, Reicherter M, Haist T and Tiziani H J (2000). Multi-functional optical tweezers using computer-generated holograms. Optics Communications 185: 77-82.

Liu S and Han M (2005). Synthesis, functionalization and bioconjugation of monodisperse silicacoated gold nanoparticles: robust bioprobes. Advanced Functional Materials 15: 961-967.

Madou M (1997). Fundamentals of microfabrication. Boca Raton, FL, CRC Press.

Malinauskas M, Farsari M, Piskarskas A and Juodkazis S (2013). Ultrafast laser nanostructuring of photopolymers: A decade of advances. Physics Reports 533: 1-31.

Marie R, Schmid S, Johansson A, Ejsing L, Nordström M, Häfliger D, Christensen C, Boisen A and Dufva M (2006). Immobilisation of DNA to polymerised SU-8 photoresist. Biosensors and Bioelectronics 21: 1327-1332.

Maruo S, Nakamura O and Kawata S (1997). Three dimensional microfabrication with twophoton-absorbed photopolymerization. Optics Letters 22: 132-134.

Matveeva E G, Gryczynski I, Barnett A, Leonenko Z, Lakowicz J R and Gryczynski Z (2007). Metal particle-enhanced fluorescent immunoassays on metal mirrors. Analytical Biochemistry 363: 239-245.

Mayer K M, Lee S, Liao H, Rostro B C, Fuentes A, Scully P T, Nehl C and Hafner J H (2008). A label-free immunoassay based upon localized surface plasmon resonance of gold nanorods. ACS Nano 2: 687-692.

McPherson T, Kidane A, Szleifer I and Park K (1998). Prevention of protein adsorption by tethered PEO Layers: experiments and single chain mean field analysis. Langmuir 14: 176-186. 
Moffitt J R, Chemla Y R, Smith S B and Bustamante C (2008). Recent advances in optical tweezers. Annual Review of Biochemistry 77: 205-228.

Mohamed M B, Volkov V, Link S and El-Sayed M A (2000). The 'lightning' gold nanorods: fluorescence enhancement of over a million compared to the gold metal. Chemical Physics Letters 317: 517-523.

Mohammad S, Jun L, Robert A F, James T I, Summer N S, Obert M F, Michal L and Michelle D W (2014). Nanophotonic trapping for precise manipulation of biomolecular arrays. Nature Nanotechnology 9: 448-452.

Neuman K C and Block S M (2004). Optical trapping. Review of Scientific Instruments 75 : 2787-2890.

Obata K, Koch J, Hinze U and Chichkov B (2010). Multi-focus two-photon polymerization technique based on individually controlled phase modulation. Optics Express 18: 1719317200 .

Oroszi L, Galajda P, Kirei H, Bottka S and P O (2006). Direct measurement of torque in an optical trap and its application to double-strand DNA. Physical Review Letters 97: 1-6.

Ostendorf A and N C B (2006). Two-photon polymerization: A new approach to micromachining. Photonics Spectra 40: 72-80.

Ovsianikov A, Gruene M, Pflaum M, Koch L, Maiorana F, Wilhelmi M, Haverich A and Chichkov B (2010). Laser printing of cells into 3D scaffolds. Biofabrication 2: 014104014111.

Ovsianikov A, Ostendorf A and Chichkov B N (2007). Three-dimensional photofabrication with femtosecond lasers for applications in photonics and biomedicine. Applied Surface Science 253: 6599-6602.

Palima D, Banas A R, Vizsnyiczai G, Kelemen L, Ormos P and Gluckstad J (2012). Wave-guided optical waveguides. Optics Express 20: 2004-2014. 
Papra A, Bernard A, Juncker D, Larsen N B, Michel B and Delamarche E ( 2001). Microfluidic networks made of poly (dimethylsiloxane), $\mathrm{Si}$, and $\mathrm{Au}$ coated with polyethylene glycol for patterning proteins onto surfaces. Langmuir 17: 4090-4095.

Plummer J, Deal M D and Griffin P (2000). Silicon VLSI Technology Fundamentals, Practice and Modeling. New Jersey Prentice-Hall.

Pollard M R, Botchway S W, Chichkov B, Freeman E, Halsall R N J, Jenkins D W K, Loader I, Ovsianikov A, Parker A W, Stevens R, Turchetta R, Ward A D and Towrie M (2010). Optically trapped probes with nanometer-scale tips for femto-Newton force measurement. New Journal of Physics 12: 113056-113070.

Pompa P P, Martiradonna L, Torre A D, Sala F D, Manna L, De Vittorio M, Calabi F, Cingolani $\mathrm{R}$ and Rinaldi R (2006). Metal-enhanced fluorescence of colloidal nanocrystals with nanoscale control. Nature Nanotechnology 1: 126-130.

Radha B, Arif M f, Datta R, Kundu T K and Kulkarni G U (2010). Movable Au microplates as fluorescence enhancing substrates for live cells. Nano Research 3: 738-747.

Raimondi M, Eaton S M, Nava M M, Laganà M, Cerullo G and Osellame R (2012). Two-photon laser polymerization: from fundamentals to biomedical application in tissue engineering and regenerative medicine. Journal of Applied Biomaterials \& Biomechanics 10: 55-65.

Raschke G, Kowarik S, Franzl T, Sönnichsen C, Klar T, Feldmann J, Nichtl A, Kürzinger, Obata K, Koch J, U H and Chichkov B (2003). Biomolecular recognition based on single gold nanoparticle light scattering. Nano Letters 3: 965-938.

Ristic L (1994). Sensor Technology and Devices. Boston, Artech House.

Ritschdorff E, Nielson R and Shear J B (2012). Multi-focal multiphoton lithography. Lab on a Chip 12: 867-871.

Rodrigo P J, Kelemen L, Alonzo C A, Perch-Nielsen I R, Dam J S, Ormos P and Glückstad J (2007). Optical manipulation and assembly of shape complementary planar microstructures. Optics Express 15: 9009-9014. 
Rodrigo P J, Kelemen L, Palima D, Alonzo C, Ormos P and Glückstad J (2009). Optical microassembly platform for constructing reconfigurable microenvironments for biomedical studies. Optics Express 17: 6578-6583.

Ruiz-Taylor L A, Martin T L, Zaugg F G, Witte K, Indermuhle P, Nock S and Wagner P ( 2001). Monolayers of derivatized poly(L-lysine)-grafted poly(ethylene glycol) on metal oxides as a class of biomolecular interfaces. Proceedings of the National Academy of Sciences U.S.A 98: 852-857.

Schizas C, Melissinaki V, Gaidukeviciute A, Reinhardt C, Ohrt C, Dedoussis V, Chichkov B N, Fotakis C, Farsari M and Karalekas D (2010). On the design and fabrication by twophoton polymerization of a readily assembled micro-valve. International Journal of Advanced Manufacturing Technology 48: 435-441.

Schlapak R, Pammer P, Armitage D, Zhu R, Hinterdorfer P, Vaupel M, Frühwirth T and Howorka S (2006). Glass surfaces grafted with high-density poly(ethylene glycol) as substrates for DNA oligonucleotide microarrays. Langmuir 22: 277-285.

Schulte T, Bardell R L and Weigl B H (2002). Microfluidic technologies in clinical diagnostics. Clinica Chimica Acta 321: 1-10.

Shaw J M, Gelorme J D, LaBianca N C, Conley W E and Holmes S J (1997). Negative photoresists for optical lithography. IBM Journal of Research and Development 41: 8194.

Shuhui W U, Jesper S and Min G U (2006). Two-photon polymerisation for three-dimensional micro-fabrication. Journal of Photochemistry and Photobiology A: Chemistry 181: 1-11.

Stadler B, Falconnet D, Pfeiffer I, Hook F and Voros J ( 2004). Micropatterning of DNA-tagged vesicles. Langmuir 20: 11348-11354.

Stranik O, McEvoy H M, McDonagh C and MacCraith B D (2005). Plasmonic enhancement of fluorescence for sensor applications. Sensors and Actuators B: Chemical 107: 148-153. 
Sun H B, Maeda M, Takada K, James W M, Chon M G and Kawata S (2003). Experimental investigation of single voxels for laser nanofabrication via two-photon photopolymerization. Applied Physics Letters 83: 819-821.

Tao S L, Popat K and Desai T A (2006). Off-wafer fabrication and surface modification of asymmetric 3D SU-8 microparticles. Nature Protocols 1: 3153-3158.

Tao S L, Popat K C, Norman J J and Desai T A (2008). Surface modification of SU-8 for enhanced biofunctionality and nonfouling properties. Langmuir 24: 2631-2636.

Trull J, Maigyte L, Mizeikis V, Malinauskas M, Juodkazis S, Cojocaru C, Rutkauskas M, Peckus M, Sirutkaitis V and Staliunas K (2011). Formation of collimated beams behind the woodpile photonic crystal. Physical Review A 84: 033812-033817.

Tseng F G, Lin K H, Hsu H T and Chieng C C (2004). A surface-tension-driven fluidic network for precise enzyme batch-dispensing and glucose detection. Sensors and Actuators A: Physical 111: 107-117.

Turkevich J, Stevenson P and Hillier J (1951). A study of the nucleation and growth processes in the synthesis of colloidal gold. Discussions of the Faraday Society 11: 55-75.

Turner M D, Schroder-Turk G and M G (2011). Fabrication and characterization of threedimensional biomimetic chiral composites. Optics Express 19: 10001-10008.

Vizsnyiczai G, Kelemen L and Ormos P (2014). Holographic multi-focus 3D two-photon polymerization with real-time calculated holograms. Optics Express 22: 24217-24223.

Voskerian G, Shive M S, Shawgo R S, von Recum H, Anderson J M, Cima M J and R L (2003). Biocompatibility and biofouling of MEMS drug delivery devices. Biomaterials 24: 19591967.

Wang Y, Pai J, Hong Lai H, Sims C, Bachman M, Li G and Allbritton N L (2007). Surface graft polymerization of SU-8 for bio-MEMS applications. Journal of Micromechanics and Microengineering 17: 1371-1380. 
Wang Z, El-Ali J, Engelund M, Gotsæd T, Perch-Nielsen I R, Mogensen K B, Snakenborg D, Kutter J P and Wolf A (2004). Measurements of scattered light on a microchip flow cytometer with integrated polymer based optical elements. Lab on a Chip 4: 372-377.

Williams M C (2002). Optical tweezers measuring piconewton forces, in single molecule techniques. Bethesda, MD, Petra Schwille, ed., a volume of the Biophysics Textbook Online.

Wong J, Chilkoti A and Moy V T ( 1999). Direct force measurements of the streptavidin -biotin interaction. Biomolecular Engineering 16: 45 - 55.

Zhang J, Fu Y, Chowdhury M H and Lakowicz J R (2007). Metal-enhanced single-molecule fluorescence on silver particle monomer and dimer: coupling effect between metal particles. Nano Letters 7: 2101-2107.

Zin M, Leong K, Wong N, Ma H, Sarikaya M and Jen A Y (2009). Surface-plasmon-enhanced fluorescence from periodic quantum dot arrays through distance control using biomolecular linkers. Nanotechnology 20: 015305-015312. 